\title{
Reconfigurable Intelligent Surface assisted Two-Way Communications: Performance Analysis and Optimization
}

\author{
Saman Atapattu, Rongfei Fan, Prathapasinghe Dharmawansa, Gongpu Wang, \\ Jamie Evans, and Theodoros A. Tsiftsis
}

\begin{abstract}
In this paper, we investigate the two-way communication between two users assisted by a re-configurable intelligent surface (RIS). The scheme that two users communicate simultaneously in the same time slot over Rayleigh fading channels is considered. The channels between the two users and RIS can either be reciprocal or non-reciprocal. For reciprocal channels, we determine the optimal phases at the RIS to maximize the signal-to-interference-plus-noise ratio (SINR). We then derive exact closed-form expressions for the outage probability and spectral efficiency for single-element RIS. By capitalizing the insights obtained from the single-element analysis, we introduce a gamma approximation to model the product of Rayleigh random variables which is useful for the evaluation of the performance metrics in multiple-element RIS. Asymptotic analysis shows that the outage decreases at $(\log (\rho) / \rho)^{L}$ rate where $L$ is the number of elements, whereas the spectral efficiency increases at $\log (\rho)$ rate at large average SINR $\rho$. For non-reciprocal channels, the minimum user SINR is targeted to be maximized. For singleelement RIS, closed-form solutions are derived whereas for multiple-element RIS the problem turns out to be non-convex. The latter is relaxed to be a semidefinite programming problem, whose optimal solution is achievable and serves as a sub-optimal solution.
\end{abstract}

Index Terms-Outage probability, reconfigurable intelligent surface (RIS), spectral efficiency, two-way communications.

\section{INTRODUCTION}

Multiple antenna systems exploit spatial diversity not only to increase throughput but also to enhance the reliability of the wireless channel. Alternatively, radio signal propagation via man-made intelligent surfaces has emerged recently as an attractive and smart solution to replace power-hungry active components [1], [2]. Such smart radio environments, that have the ability of transmitting data without generating new radio waves but reusing the same radio waves, can thus be implemented with the aid of reflective surfaces. This novel concept utilizes electromagnetically controllable surfaces that can be integrated into the existing infrastructure, for example, along

S. Atapattu and J. Evans are with the Department of Electrical and Electronic Engineering, The University of Melbourne, Victoria, Australia (emails: \{saman.atapattu, jse\}@unimelb.edu.au).

R. Fan is with the School of Information and Electronics, Beijing Institute of Technology, Beijing 100081, P. R. China (email: fanrongfei@bit.edu.cn).

P. Dharmawansa is with Department of Electronic and Telecommunications Engineering, University of Moratuwa, Moratuwa, Sri Lanka (email: prathapa@uom.lk).

G. Wang is with the School of Computer and Information Technology, Beijing Jiaotong University, Beijing 100044, P. R. China (email: gpwang@bjtu.edu.cn)

Theodoros A. Tsiftsis is with the Institute of Physical Internet and the School of Intelligent Systems Science and Engineering, Jinan University, Zhuhai 519070, China (e-mail: theo_tsiftsis@jnu.edu.cn). the walls of buildings. Such a surface is frequently referred to as Reconfigurable Intelligent Surface (RIS), Large Intelligent Surface (LIS) or Intelligent Reflective Surface (IRS). Its tunable and reconfigurable reflectors are made of passive or almost passive electromagnetic devices which exhibit a negligible energy consumption compared to the active elements or nodes. For instance, the RIS-assisted communication outperforms the conventional relaying techniques in terms of energy efficiency. Since the energy efficiency in turn is a function of data rate, power consumption and frequency/time resource usage, this significant efficiency improvement with RIS can address several major issues arising from future wireless applications such as increasing demand for data rates, spectrum crunch, high energy consumption and environment impact.

This brand-new concept has already been proposed to incorporated into various wireless techniques - multi-cell multipleinput multiple-output (MIMO) systems [3], massive MIMO [4], non-orthogonal multiple access (NOMA) [5], energy harvesting [6], optical communications [7] to name a few. The RIS can make the radio environment smart by collaboratively adjusting the phase shifts of reflective elements in real time. This results in the desired signals being constructively interfered at the receiver, whereas and other signals being interfered destructively. Therefore, most existing work on RIS focus on phase optimization of RIS elements [2], [8]-[15]. However, there are very limited research efforts explored the communication-theoretic performance limits [13], [16]-[19]. The remainder of this section has an overview of related work, followed by a summary on contributions of this work.

\section{A. Related Work}

An RIS-enhanced point-to-point multiple-input singleoutput (MISO) system is considered in [8], which aims to maximize the total received signal power at the user by jointly optimizing the (active) transmit beamforming at the access point and (passive) reflect beamforming at RIS. The authors propose a centralized algorithm based on the technique of semi-definite relaxation (SDR) by assuming the availability of global channel state information (CSI) at the RIS. A similar system model is also considered in [9] where the beamformer at the access point and the RIS phase shifts are jointly optimized to maximize the spectral efficiency. The resultant non-convex problem is solved with the help of fixed point iteration and manifold optimization techniques. An RIS-enhanced orthogonal frequency division multiplexing 
(OFDM) system under frequency-selective channels is considered in [10]. For the proposed sub-carrier grouping method for channel estimation, the achievable rate is maximized by jointly optimizing the transmit power allocation and the RIS passive array reflection coefficients. This non-convex problem is subsequently solved sub-optimally by alternately optimizing the power and array coefficients in an iterative manner. For a phase dependent amplitude in the reflection coefficient, in [11], the transmit beamforming and the RIS reflect beamforming are jointly optimized based on an alternating optimization technique to achieve a low-complex sub-optimal solution. For downlink multi-user communication helped by RIS from a multi-antenna base station, both the transmit power allocation and the phase shifts of the reflecting elements are designed to maximize the energy efficiency on subject to individual link budget in [2]. There the authors use gradient descent search and sequential fractional programming to solve the resultant non-convex problem. The weighted sum-rate of all users is maximized by joint optimizing the active beamforming at the base-station and the passive beamforming at the RIS for multiuser MISO systems in [12]. The resultant non-convex problem is first decoupled via Lagrangian dual transform, and then the beamforming vectors are optimized alternatively. Moreover, user fairness is considered for a LIS-aided downlink of a single-cell multi-user system in [13], whereas physical layer security issues are considered in [14], [15].

As mentioned before, while the optimization of power and/or phase shift have received more attention in recent work, a few have focused on analytical performance evaluation. Therefore, very limited number of results are available so far in this respect. For an LIS-assisted large-scale antenna system, an upper bound on the ergodic capacity is first derived and then a procedure for phase shift design based on the upper bound is discussed in [16]. In [13], an optimal precoding strategy is proposed when the line-of-sight (LoS) channel between the base station and and the LIS is of rank-one, and some asymptotic results are also derived for the LoS channel of rank-two and above. An asymptotic analysis of the data rate and channel hardening effect in an LIS-based large antenna-array system is presented in [17] where the estimation errors and interference are taken into consideration. For a large RIS system, some theoretical performance limits are also explored in [18] where the symbol error probability is derived by characterizing the receive SNR using the central limit theorem (CLT). In [19], the LIS transmission with phase errors is considered and the composite channel is shown to be equivalent to a point-to-point Nakagami fading channel. Subsequent performance analysis of the system has been conducted based on this equivalent channel model.

On the other hand, two-way communications exchange messages of two or more users over the same shared channel [20]. Since this improves the spectral efficiency of the system, two-way techniques will have a significant impact on current and next generation cellular networks applications such as mobile video conferencing, communication between a base station and clients, and device-to-device communications. While the two-way network provides full-duplex type information exchange for the point-to-point or D2D communica- tions, it also enables maximum spectral efficiency for relaying network with a full-duplex relay node [21]. The benefits of two-way network are contingent on proper self-interference cancellation, which is possible with the recent signal processing breakthroughs. Therefore, two-way communications have been recently attracted considerable attention, and have already been thoroughly investigated with respect to most of the novel $4 \mathrm{G}$ and $5 \mathrm{G}$ wireless technologies such as massive MIMO, full-duplex communications, NOMA, mmWave communications, and cognitive radio, to mention but a few [22][24]. Thus, the RIS may also serve as a potential candidate for further performance improvement in the two-way Beyond $5 \mathrm{G}$ or $6 \mathrm{G}$ systems. However, to the best of our knowledge, all these previous work on RIS considered the one-way communications. Motivated by this reason, as the first work, we study the RIS for two-way communications in view of quantifying the performance limits, which is the novelty of this paper.

\section{B. Summary of Contributions}

Generally speaking, although the RIS can introduce a delay, it may be negligible compared to the actual data transmission time duration. Therefore, the transmission protocol and analytical model of the RIS-assisted two-way communication may differ from the traditional relay-assisted two-way communications. Fig. 1 summarizes two possible RIS-assisted transmission schemes which require different number of time slots to achieve the bi-directional data exchange between two users.

- Scheme 1 (one time-slot transmission): As shown in Fig. 1a, two end-users simultaneously transmit their own data to the RIS which reflects received signal with negligible delay. Since the signal is received without delay, each end-user should be implemented with a pair of antennas each for signal transmission and reception, where each user experiences a full-duplex type communication as well.

- Scheme 2 (two time-slots transmission): As shown in Fig. 1b, the user 1 transmits its data to the user 2 in the first time slot, and vice versa in the second time slot. Therefore, each end-user may use a single antenna for signal transmission and reception.

Since Scheme 1 is more exciting and interesting; and also Scheme 2 can be deduced from Scheme 1, we develop our analytical framework based on Scheme 1. In Scheme 1, concurrent transmissions occur from the user-to-RIS and the RIS-to-user. We consider both cases where user-to-RIS and RIS-to-user channels are assumed to be reciprocal and nonreciprocal.

Although the RIS may be implemented with large number of reflective elements for the future wireless networks, fundamental communication-theoretic foundations for single and moderate number of elements of the RIS have not been wellunderstood under multi-path fading. However, such knowledge is very critical for network design. As cell-free massive MIMO is a promising extension to co-located massive MIMO, another further research direction of the RIS will be a distributed RIS 


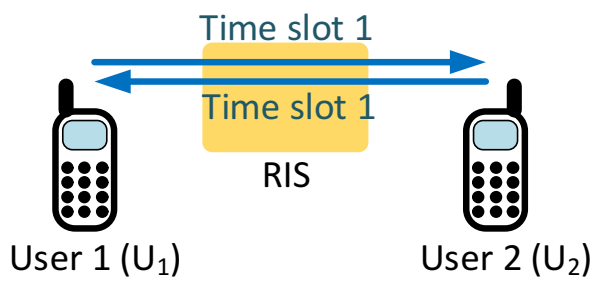

(a) Scheme 1: One time-slot transmission.

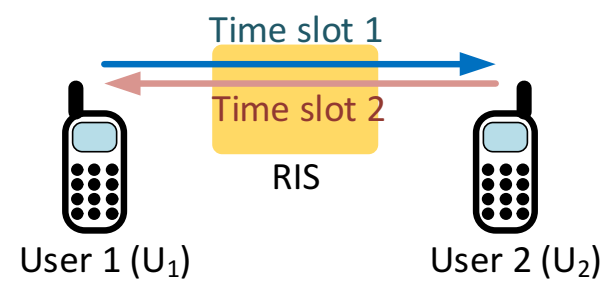

(b) Scheme 2: Two time-slot transmission.

Fig. 1: Two possible transmission schemes for two-way communications.

system. Such system design is based on the understanding of a simple RIS system where each distributed RIS may have single or very few reflective elements. Further, the end-toend SINR expression is different from the SINR expression of either the conventional amplify-and-forward (AF) relaying or the instantaneous relaying because these relay types are implemented with active elements which need proper power control at relaying stage [25]. Therefore, we need new analytical frameworks to evaluate the performance for both singleelement and multiple-element RIS cases.

To support the aforementioned research directions, this paper analyzes a general two-way RIS system where the number of reflective elements can range from one to any arbitrary value, and provides several communication-theoretic properties which have not been well-understood yet.

The main contributions of the paper are summarized as follows:

1) For reciprocal channels with a single-element RIS, we first derive the exact outage probability and spectral efficiency in closed-form for the optimal phase adjustment at the RIS. We then provide asymptotic results for sufficiently large transmit power compared to the noise and interference powers. Our analysis reveals that the outage decreases at $\log (\rho) / \rho$ rate, whereas the spectral efficiency increases at $\log (\rho)$ rate for asymptotically large signal-to-interference-plus-noise ratio (SINR), $\rho$.

2) For reciprocal channels with a multiple-element RIS, where the number of elements, $L$, is more than one but not necessarily as large as in LIS. In this respect, the instantaneous SINR turns out to take the form of a sum of product of two Rayleigh random variables (RVs). As well documented in the literature, this does not admit a tractable PDF or CDF expression. To circumvent this problem, we first approximate the product of two Rayleigh RVs with a Gamma RV, and then evaluate the outage probability and spectral efficiency. This seems to be the first paper which uses gamma approximation for a Rayleigh product. Surprisingly, this approximation works well and more accurately than the CLT approximation (which is frequently used in LIS literature), even for a moderate number of elements such as $L=32$ or $L=64$. Since the tail of the gamma approximation does not follow the exact distribution of the SINR RV, we resort to asymptotic analysis of the exact SINR for single-element RIS. In this respect. we show that the outage decreases at $(\log (\rho) / \rho)^{L}$ rate, whereas the spectral efficiency still increases at $\log (\rho)$ rate.

3) For non-reciprocal channels, system performance analysis seems an arduous task, since four different channel phases are involved. In this case, we turn to optimize the phase so as to maximize an important measure: the minimum user SINR, which represents user fairness. With single-element RIS, closed-form solution is derived. However, for multiple-element RIS, the associated problem is non-convex. To find the solution, through some transformations, we relax the formulated problem to be a semidefinite programming (SDP) problem, the optimal solution of which is achievable and can further render a sub-optimal solution for our originally formulated optimization problem.

Overall, this paper attempts to strike the correct balance between the performance analysis and optimization of twoway communications with the RIS.

Before proceeding further, here we introduce a list of symbols that have been used in the manuscript. We use lowercase and uppercase boldface letters to denote vectors and matrices respectively. A complex Gaussian random variable $X$ with zero mean and variance $\sigma^{2}$ is denoted by $X \sim \mathcal{C N}\left(\mu, \sigma^{2}\right)$, whereas a real Gaussian random variable is denoted by $X \sim$ $\mathcal{N}\left(0, \sigma^{2}\right)$. The magnitude of a complex number $z$ is denoted by $|z|$ and $\mathrm{E}[\cdot]$ represents the mathematical expectation operator.

\section{SySTEM MODEL}

A RIS-aided two-way wireless network that consists of two end users (namely, $U_{1}$ and $U_{2}$ ) and a reflective surface $(R)$ where the two-way networks with reciprocal and nonreciprocal channels are shown in Fig. 2a and Fig. 2b, respectively. The two users exchange their information symbols concurrently via the passive RIS, which only adjusts the phases of incident signals. Each user is equipped with a pair of antennas for the transmission and reception. The RIS contains $L$ reconfigurable reflectors where the $\ell$ th passive element is denoted as $I_{\ell}$. No direct link between two users is assumed, due to transmit power limitation or severe shadowing effect. For simplicity, we assume that both users use the same codebook. The unit-energy information symbols from $U_{1}$ and $U_{2}$, randomly selected from the codebook, are denoted by $s_{1}$ and $s_{2}$, respectively. The power budgets are $P_{1}$ and $P_{2}$ for end users $U_{1}$ and $U_{2}$, respectively. We assume that all fading channels are independent. By placing the antennas of users and 


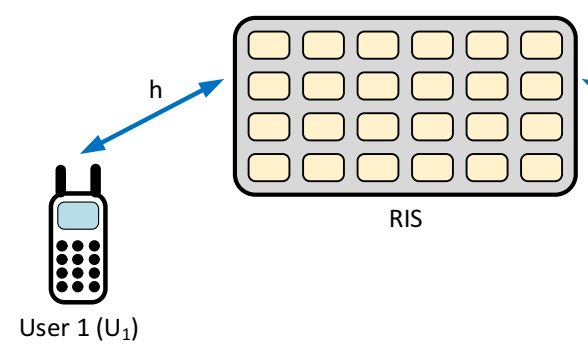

(a) With reciprocal channels.

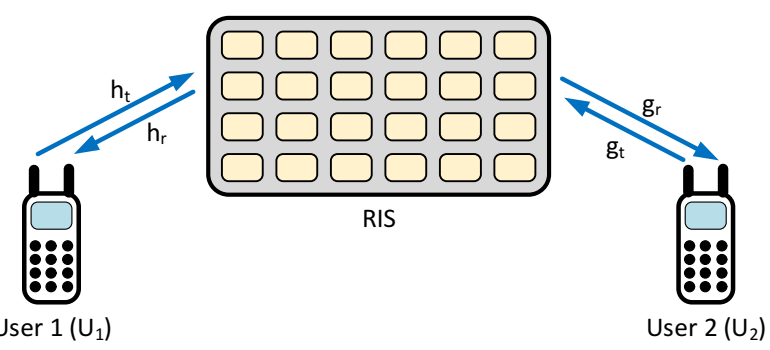

(b) With non-reciprocal channels.

Fig. 2: Two-way communications via RIS.

elements of RIS sufficiently apart, the channel gains between different antenna pairs fade more or less independently and no correlation exist.

\section{A. Reciprocal Channels}

The wireless channel can be assumed to be reciprocal if the overall user-to-RIS and RIS-to-user transmission time falls within a coherence interval of the channel and the pair of antennas are placed sufficiently close distance, see Fig. 2a.

In this case, we denote the fading coefficients from $U_{1}$ to the $I_{\ell}$ and from $U_{2}$ to the $I_{\ell}$ as $h_{\ell}=\alpha_{\ell} \mathrm{e}^{-j \varphi_{\ell}}$ and $g_{\ell}=$ $\beta_{\ell} \mathrm{e}^{-j \psi_{\ell}}$, respectively. The channels are reciprocal such that the channels from the $I_{\ell}$ to the two end users are also $h_{\ell}$ and $g_{\ell}$, respectively. All channels are assumed to be independent and identically distributed (i.i.d.) complex Gaussian fading with zero-mean and $\sigma^{2}$ variance, i.e., $h_{\ell}, g_{\ell} \sim \mathcal{C N}\left(0, \sigma^{2}\right)$. Therefore, magnitudes of $h_{\ell}$ and $g_{\ell}$ (i.e., $\alpha_{\ell}$ and $\beta_{\ell}$ ) follow the Rayleigh distribution. It is assumed that the two end users know all channel coefficients, $h_{1}, \ldots, h_{L}$ and $g_{1}, \ldots, g_{L}$, and the $I_{\ell}$ knows its own channels' phase values $\varphi_{\ell}$ and $\psi_{\ell}$. This channel information requirement can be satisfied in advance by using compressive sensing or deep learning techniques [26].

Each user receives a superposition of the two signals via the RIS. Thus, the receive signal at $U_{1}$ at time $t$ can be given as

$$
\begin{aligned}
y_{1}(t)= & \underbrace{\sqrt{P_{2}}\left(\sum_{\ell=1}^{L} g_{\ell} \mathrm{e}^{j \phi_{\ell}} h_{\ell}\right) s_{2}(t)}_{\text {Desired signal }}+\underbrace{i_{1}(t)}_{\text {Loop interference }} \\
& +\underbrace{\sqrt{P_{1}}\left(\sum_{\ell=1}^{L} h_{\ell} \mathrm{e}^{j \phi_{\ell}} h_{\ell}\right) s_{1}(t)}_{\text {Self interference }}+\underbrace{w_{1}(t)}_{\text {Noise }}
\end{aligned}
$$

where $\phi_{\ell}$ is the adjustable phase induced by the $I_{\ell}, i_{1}(\tau)$ is the receive residual self-interference resulting from several stages of cancellation and $w_{1}(t)$ is the additive white Gaussian noise (AWGN) at $U_{1}$ which is assumed to be i.i.d. with distribution $\mathcal{C N}\left(0, \sigma_{w_{1}}^{2}\right)$. Further, the vectors of channel coefficients between the two users and RIS are given as $\mathbf{h}=\left[h_{1}, \cdots, h_{L}\right]^{\mathrm{T}}$ and $\mathbf{g}=\left[g_{1}, \cdots, g_{L}\right]^{\mathrm{T}}$. The phase shifts introduced by the RIS are given by a diagonal matrix as $\boldsymbol{\Phi}=\operatorname{diag}\left(\left[\mathrm{e}^{j \phi_{1}}, \cdots, \mathrm{e}^{j \phi_{L}}\right]\right)$. Then, we can write (1) as

$y_{1}(t)=\sqrt{P_{2}} \mathbf{h}^{\mathrm{T}} \boldsymbol{\Phi} \mathbf{g} s_{2}(t)+\sqrt{P_{1}} \mathbf{h}^{\mathrm{T}} \boldsymbol{\Phi} \mathbf{h} s_{1}(t)+i_{1}(t)+w_{1}(t)$,

where $\sqrt{P} \mathbf{h}^{\mathrm{T}} \boldsymbol{\Phi} \mathbf{h} s_{1}(t)$ denotes the self interference term. Since the $U_{1}$ has the global CSI, it can completely eliminate the self-interference. Therefore, after the elimination, the received instantaneous SINR at $U_{1}$ can be written as

$$
\gamma_{1}=\frac{\left|\sqrt{P_{2}}\left(\sum_{\ell=1}^{L} g_{\ell} \mathrm{e}^{j \phi_{\ell}} h_{\ell}\right) s_{2}(t)\right|^{2}}{\left|i_{1}(t)\right|^{2}+\left|w_{1}(t)\right|^{2}} .
$$

To avoid loop interference, similar to full-duplex communications, the $U_{1}$ applies some sophisticated loop interference cancellations, which results in residual interference [27]. Among different models used in the literature for full-duplex communications, in this paper, we adopt the model where $i_{1}(t)$ is i.i.d. with zero-mean, $\sigma_{i_{1}}^{2}$ variance, additive and Gaussian, which has similar effect as the AWGN [27]. Further, the variance is modeled as $\sigma_{i_{1}}^{2}=\omega P_{1}^{\nu}$ for $P_{1} \geq 1$, where the two constants, $\omega>0$ and $\nu \in[0,1]$, depend on the cancellation scheme used at the user. Thus, the instantaneous SINR at $U_{1}$ in (3) can be simplified as

$$
\gamma_{1}=\frac{P_{2}\left|\sum_{\ell=1}^{L} \alpha_{\ell} \beta_{\ell} \mathrm{e}^{j\left(\phi_{\ell}-\varphi_{\ell}-\psi_{\ell}\right)}\right|^{2}}{\sigma_{i_{1}}^{2}+\sigma_{w_{1}}^{2}} .
$$

Similarly, we can write the instantaneous SINR at $U_{2}$ as

$$
\gamma_{2}=\frac{P_{1}\left|\sum_{\ell=1}^{L} \alpha_{\ell} \beta_{\ell} \mathrm{e}^{j\left(\phi_{\ell}-\varphi_{\ell}-\psi_{\ell}\right)}\right|^{2}}{\sigma_{i_{2}}^{2}+\sigma_{w_{2}}^{2}} .
$$

where $\sigma_{w_{2}}^{2}$ is the noise variance and $\sigma_{i_{2}}^{2}$ is the variance of residual interference at the $U_{2}$. It can also be modeled as $\sigma_{i_{2}}^{2}=$ $\omega P_{2}^{\nu}$.

\section{B. Non-Reciprocal Channels}

Even though the overall user-to-RIS and RIS-to-user transmission time falls within a coherence interval of the channel, the wireless channel can be assumed to be non-reciprocal when the pair of antennas are implemented far apart each other or non-reciprocal hardware for transmission and reception, see Fig. 2b. 
In this case, the fading coefficients from the transmit antenna of $U_{1}$ to the $I_{\ell}$ and from the $I_{\ell}$ to the receive antenna of $U_{1}$ are denoted as $h_{\mathrm{t}, \ell}=\alpha_{\mathrm{t}, \ell} \mathrm{e}^{-j \varphi_{\mathrm{t}, \ell}}$ and $h_{\mathrm{r}, \ell}=\alpha_{\mathrm{r}, \ell} \mathrm{e}^{-j \varphi_{\mathrm{r}, \ell}}$, where $\alpha_{\mathrm{t}, \ell}, \alpha_{\mathrm{r}, \ell}, \varphi_{\mathrm{t}, \ell}$ and $\varphi_{\mathrm{r}, \ell}$ denote amplitudes and phases, respectively. Similarly, the respective channels associated with the $U_{2}$ are denoted as $g_{\mathrm{t}, \ell}=\beta_{\mathrm{t}, \ell} \mathrm{e}^{-j \psi_{\mathrm{t}, \ell}}$ and $g_{\mathrm{r}, \ell}=\beta_{\mathrm{r}, \ell} \mathrm{e}^{-j \psi_{\mathrm{r}, \ell}}$. All channels are assumed to be independent and identically distributed (i.i.d.) complex Gaussian with zero-mean and $\sigma^{2}$ variance (i.e., $\left.h_{\mathrm{t}, \ell}, h_{\mathrm{r}, \ell}, g_{\mathrm{t}, \ell}, g_{\mathrm{r}, \ell} \sim \mathcal{C N}\left(0, \sigma^{2}\right)\right)$. It is assumed that the two end users have full CSI knowledge, i.e., $\mathbf{h}_{\mathrm{t}}=$ $\left[h_{\mathrm{t}, 1}, \ldots, h_{\mathrm{t}, L}\right], \mathbf{h}_{\mathrm{r}}=\left[h_{\mathrm{r}, 1}, \ldots, h_{\mathrm{r}, L}\right], \mathbf{g}_{\mathrm{t}}=\left[g_{\mathrm{t}, 1}, \ldots, g_{\mathrm{t}, L}\right]$ and $\mathbf{g}_{\mathrm{r}}=\left[g_{\mathrm{r}, 1}, \ldots, g_{\mathrm{r}, L}\right]$; and each $I_{\ell}$ element knows its own channels' phases, i.e., $\varphi_{\mathrm{t}, \ell}, \varphi_{\mathrm{r}, \ell}, \psi_{\mathrm{t}, \ell}$ and $\psi_{\mathrm{r}, \ell}$.

Thus, the receive signal at $U_{1}$ at time $t$ can be written as

$$
\begin{aligned}
y_{1}(t)= & \sqrt{P_{2}}\left(\sum_{\ell=1}^{L} h_{\mathrm{r}, \ell} \mathrm{e}^{j \phi_{\ell}} g_{\mathrm{t}, \ell}\right) s_{2}(t)+i_{1}(t) \\
& +\sqrt{P_{1}}\left(\sum_{\ell=1}^{L} h_{\mathrm{r}, \ell} \mathrm{e}^{j \phi_{\ell}} h_{\mathrm{t}, \ell}\right) s_{1}(t)+w_{1}(t),
\end{aligned}
$$

where $\sqrt{P_{1}}\left(\sum_{\ell=1}^{L} h_{\mathrm{r}, \ell} \mathrm{e}^{j \phi_{\ell}} h_{\mathrm{t}, \ell}\right) s_{1}(t)$ denotes the self interference, which can be eliminated due to global CSI,, and thus, subsequently, self-interference cancellation can be applied. We assume the same statistical properties for self-interference as in (1) for comparison purposes. Then, the SINR at $U_{1}$ can be written as

$$
\gamma_{1}=\frac{P_{2}\left|\sum_{\ell=1}^{L} \alpha_{\mathrm{r}, \ell} \beta_{\mathrm{t}, \ell} \mathrm{e}^{j\left(\phi_{\ell}-\varphi_{\mathrm{r}, \ell}-\psi_{\mathrm{t}, \ell}\right)}\right|^{2}}{\sigma_{i_{1}}^{2}+\sigma_{w_{1}}^{2}} .
$$

By performing the similar signal processing techniques as in $U_{1}$, the SINR of $U_{2}$ can be written as

$$
\gamma_{2}=\frac{P_{1}\left|\sum_{\ell=1}^{L} \beta_{\mathrm{r}, \ell} \alpha_{\mathrm{t}, \ell} \mathrm{e}^{j\left(\phi_{\ell}-\psi_{\mathrm{r}, \ell}-\varphi_{\mathrm{t}, \ell}\right)}\right|^{2}}{\sigma_{i_{2}}^{2}+\sigma_{w_{2}}^{2}}
$$

where $\sigma_{w_{2}}^{2}$ is the noise variance and $\sigma_{i_{2}}^{2}$ is the variance of residual self-interference at the $U_{2}$.

\section{Network With Reciprocal Channels}

\section{A. Optimum Phase Design at RIS}

A careful inspection of the structures of $\gamma_{1}$ and $\gamma_{2}$ given in (4) and (5) reveals that the optimal $\phi_{\ell}$, which maximizes the instantaneous SINR of each user, admits the form

$$
\phi_{\ell}^{\star}=\varphi_{\ell}+\psi_{\ell} \text { for } \ell=1, \cdots, L .
$$

This is usually feasible at the RIS as it has the global phase information of the respective channels. Now with the aid of (4) and (5), the maximum SINRs at $U_{1}$ and $U_{2}$ can be given as $\gamma_{1}^{\star}$ and $\gamma_{2}^{\star}$, respectively, where

$$
\begin{aligned}
\gamma_{1}^{\star} & =\frac{P_{2}}{\sigma_{i_{1}}^{2}+\sigma_{w_{1}}^{2}}\left(\sum_{\ell=1}^{L} \alpha_{\ell} \beta_{\ell}\right)^{2} \\
\gamma_{2}^{\star} & =\frac{P_{1}}{\sigma_{i_{2}}^{2}+\sigma_{w_{2}}^{2}}\left(\sum_{\ell=1}^{L} \alpha_{\ell} \beta_{\ell}\right)^{2} .
\end{aligned}
$$

In general, the instantaneous SINR of each user can be written as

$$
\gamma=\rho\left(\sum_{\ell=1}^{L} \zeta_{\ell}\right)^{2}
$$

where

$$
\zeta_{\ell}=\alpha_{\ell} \beta_{\ell} \quad \text { and } \quad \rho=\left\{\begin{array}{ll}
\frac{P_{2}}{\sigma_{i_{1}}^{2}+\sigma_{w_{1}}^{2}} & \text { for } U_{1} \\
\frac{P_{1}}{\sigma_{i_{2}}^{2}+\sigma_{w_{2}}^{2}} & \text { for } U_{2}
\end{array} .\right.
$$

Further, we define $\rho$ as the average SINR, and without loss of generality, we assume $\sigma_{i_{1}}^{2}=\sigma_{i_{2}}^{2}=\sigma_{i}^{2}$ and $\sigma_{w_{1}}^{2}=\sigma_{w_{2}}^{2}=\sigma_{w}^{2}$.

\section{B. Outage Probability}

By definition, the outage probability of each user can be expressed as $P_{\text {out }}=\operatorname{Pr}\left[\gamma \leq \gamma_{\text {th }}\right]$, where $\gamma_{\text {th }}$ is the SINR threshold. This in turn gives us the important relation

$$
P_{\text {out }}=F_{\gamma}\left(\gamma_{\mathrm{th}}\right)
$$

where $F_{\gamma}(x)$ is the cumulative distribution function (CDF) of $\gamma$. To evaluate the average spectral efficiency, we need the distributions of the $\operatorname{RV} \gamma$. For general case, $\operatorname{RV} \gamma$ is a summation of $L$ independent RVs each of which is a product of two independent Rayleigh RVs. Since the analysis of the general case may give rise to some technical difficulties, we evaluate the average spectral efficiency for $L=1$ and $L \geq 2$ cases separately.

1) When $L=1$ : In this case, the instantaneous SINR of each user is $\gamma=\rho \zeta_{1}^{2}=\rho\left(\alpha_{1} \beta_{1}\right)^{2}$. Since $\alpha_{1}$ and $\beta_{1}$ are identical Rayleigh RVs with parameter $\sigma$, the PDF and CDF expressions can be written as $f_{X}(x)=\left(2 x / \sigma^{2}\right) \mathrm{e}^{-x^{2} / \sigma^{2}}$ and $F_{X}(x)=1-\mathrm{e}^{-x^{2} / \sigma^{2}}$, respectively. Since the $\mathrm{RV} \zeta_{1}$ is a product of two i.i.d. Rayleigh RVs, its CDF can be derived as $F_{\zeta_{1}}(t)=\operatorname{Pr}\left(\zeta_{1} \leq t\right)=\operatorname{Pr}\left(\alpha_{1} \leq \frac{t}{\beta_{1}}\right)$, from which we obtain

$$
F_{\zeta_{1}}(t)=\int_{0}^{\infty} F_{\alpha_{1}}\left(\frac{t}{x}\right) f_{\beta_{1}}(x) \mathrm{d} x=1-\frac{2 t}{\sigma^{2}} \mathrm{~K}_{1}\left(\frac{2 t}{\sigma^{2}}\right)
$$

where the last equality results from $\int_{0}^{\infty} \mathrm{e}^{-\frac{b}{4 x}-a x} \mathrm{~d} x=$ $\sqrt{\frac{b}{a}} \mathrm{~K}_{1}(\sqrt{a b})$ with $\mathrm{K}_{n}(\cdot)$ denoting the modified Bessel function of the second kind [28, eq. 3.324.1]. For a RV $Y=a X^{2}$ with $a>0, X \geq 0$, we can write its $\mathrm{CDF}$ as $F_{Y}(y)=$ $F_{X}(\sqrt{y / a})$. By using this fact, the CDF of $\gamma=\rho \zeta_{1}$ can be derived as

$$
F_{\gamma}(t)=1-\frac{2}{\sigma^{2}} \sqrt{\frac{t}{\rho}} \mathrm{K}_{1}\left(\frac{2}{\sigma^{2}} \sqrt{\frac{t}{\rho}}\right) .
$$

Thus, the outage probability can be written as

$$
P_{\text {out } \mid \mathrm{L}=1}\left(\gamma_{\mathrm{th}}\right)=1-\frac{2}{\sigma^{2}} \sqrt{\frac{\gamma_{\mathrm{th}}}{\rho}} \mathrm{K}_{1}\left(\frac{2}{\sigma^{2}} \sqrt{\frac{\gamma_{\mathrm{th}}}{\rho}}\right) .
$$


2) When $L \geq 2$ : In this case, the instantaneous SINR of each user is given in (12). Let us now focus on deriving the $\mathrm{CDF}$ of the $\operatorname{RV} \zeta=\sum_{\ell=1}^{L} \zeta_{\ell}$. However, by using the exact $\mathrm{CDF}$ of $\zeta_{\ell}$ given in (14), an exact statistical characterization of the $\mathrm{CDF} \zeta$ seems an arduous task. To circumvent this difficulty, in what follows we first seek an approximation for the PDF and $\mathrm{CDF}$ of $\zeta_{\ell}$.

Among different techniques of approximating distributions [29], the moment matching technique is a popular one. In the existing literature, the regular Gamma distribution is commonly used to approximate some complicated distributions because it has freedom of tuning two parameters: 1) the shape parameter $k$; and 2) the scale parameter $\theta$. The mean and variance of such Gamma distribution are $k \theta$ and $k \theta^{2}$, respectively. The following Lemma gives the Gamma approximation for the $\mathrm{CDF} F_{\zeta_{1}}(t)$.

Lemma 1. The distribution of the product of two i.i.d. Rayleigh RVs with parameter $\sigma$ can be approximated with a Gamma distribution which has the CDF

$$
F_{\zeta_{\ell}}(t) \approx \frac{1}{\Gamma(k)} \gamma\left(k, \frac{t}{\theta}\right)
$$

where

$$
k=\frac{\pi^{2}}{\left(16-\pi^{2}\right)} \text { and } \theta=\frac{\left(16-\pi^{2}\right) \sigma^{2}}{4 \pi} .
$$

Further, $\gamma(\cdot, \cdot)$ is the lower incomplete gamma function [28]. Note that, by definition, the lower and upper incomplete gamma functions satisfy $\Gamma(a, x)+\gamma(a, x)=\Gamma(a)$.

Proof: Since the first and second moments of $\zeta_{\ell}$ in (14) are $\mathrm{E}\left[\zeta_{\ell}\right]=\pi \sigma^{2} / 4$ and $\mathrm{E}\left[\zeta_{\ell}^{2}\right]=\sigma^{4}$, the $\mathrm{RV} \zeta_{\ell}$ has $\pi \sigma^{2} / 4$ mean and $\left(16-\pi^{2}\right) \sigma^{4} / 16$ variance. By matching the mean and variance of the $\mathrm{RV} \zeta_{\ell}$ with the $k \theta$ mean and $k \theta^{2}$ variance of the regular Gamma distribution, we have the above CDF.

Here we assess the accuracy of the approximation using the Kullback-Leibler (KL) divergence. In particular, we consider the KL divergence between the exact PDF of $\zeta_{\ell}$ and its approximated PDF which is defined as $\mathcal{D}_{\mathrm{KL}}=\mathrm{E}\left[\log \frac{f_{\mathrm{Exx}}(t)}{f_{\mathrm{App}}(t)}\right]$ [29] where the expectation is taken with respect to the exact probability density function (PDF) of $\zeta_{\ell}$ which can be derived as $f_{\mathrm{Ext}}(t)=4 t K_{0}\left(2 t / \sigma^{2}\right) / \sigma^{4}$. With the aid of [30, Eq. 2.16.2.2 and 2.16.20.1], this can be calculated as

$$
\begin{aligned}
\mathcal{D}_{\mathrm{KL}}=\frac{\pi \sigma^{2}}{4 \theta} & +k \ln \left(\frac{\theta}{\sigma^{2}}\right)+\epsilon(k-2) \\
& +\ln (4 \Gamma(k))+\mathrm{E}\left[\ln K_{0}\left(\frac{2 t}{\sigma^{2}}\right)\right],
\end{aligned}
$$

where $\epsilon$ is the is Euler's constant.

With numerical calculation, we plot the KL divergence vs $\sigma$ for $\sigma \in(0.05,25)$ in Fig. 3a. We get $\mathcal{D}_{\mathrm{KL}} \approx 2.3 \times 10^{-4}$ where this very small value confirms the accuracy of the approximation. Numerical result also clarifies that $\sigma$ has a little impact on $\mathcal{D}_{\mathrm{KL}}$. Moreover, Fig. 3b plots the complementary cumulative distribution function (CCDF) of $\zeta_{\ell}$ based on the simulation, the exact CDF in (14) and the approximate CDF in (17) for $\sigma^{2}=0.1,1.0,10$ which represent very small, moderate and large variance values. The exact CCDF match tightly with the Gamma approximation for the simulated $t$ range for all $\sigma^{2}$, confirming the validity of the approximation. The accuracy of the approximation is also shown by the performance curves in Section V.

Recalling that the instantaneous SINR is $\gamma=\rho\left(\sum_{\ell=1}^{L} \zeta_{\ell}\right)^{2}$ which admits the alternative decomposition

$$
\gamma=\rho \zeta^{2} \quad \text { where } \quad \zeta=\sum_{\ell=1}^{L} \zeta_{\ell} .
$$

Armed with the above lemma, now we are in a position to derive an approximate average spectral efficiency expression pertaining to the case $L \geq 2$. It is worth mentioning here that the $\mathrm{RV} \zeta$ is then a sum of $L$ i.i.d. Gamma RVs with the parameters $k$ and $\theta$. Therefore, the $\operatorname{RV} \zeta$ also follows a Gamma distribution with $L k$ and $\theta$ parameters. By using the similar variable transformation as in (15), the CDF of $\gamma$ can be approximated as

$$
F_{\gamma}(t)=\frac{1}{\Gamma(L k)} \gamma\left(L k, \frac{1}{\theta} \sqrt{\frac{t}{\rho}}\right)
$$

Therefore, the outage probability can be written as

$$
P_{\mathrm{out} \mid \mathrm{L} \geq 2}\left(\gamma_{\mathrm{th}}\right) \approx \frac{1}{\Gamma(L k)} \gamma\left(L k, \frac{1}{\theta} \sqrt{\frac{\gamma_{\mathrm{th}}}{\rho}}\right)
$$

\section{Spectral Efficiency}

The spectral efficiency can be expressed as $\log _{2}(1+\mathrm{SINR})[\mathrm{bits} / \mathrm{sec} / \mathrm{Hz}]$. Then, the average value can be evaluated as $\mathrm{R}=\int_{0}^{\infty} \log _{2}(1+x) f_{\gamma}(x) \mathrm{d} x$ where $f_{\gamma}(x)$ is the PDF of $\gamma$. By employing integration by parts, $\mathrm{R}$ can be evaluated as

$$
\mathrm{R}=\frac{1}{\log (2)} \int_{0}^{\infty} \frac{1-F_{\gamma}(x)}{1+x} \mathrm{~d} x[\mathrm{bits} / \mathrm{sec} / \mathrm{Hz}]
$$

1) When $L=1$ : With the aid of (23) and (15), the average spectral efficiency can be evaluated as

$$
\begin{aligned}
\mathrm{R}_{\mathrm{L}=1}(\rho) & =\frac{1}{\log (2)} \frac{2}{\sigma^{2} \sqrt{\rho}} \int_{0}^{\infty} \frac{\sqrt{x}}{(1+x)} \mathrm{K}_{1}\left(\frac{2}{\sigma^{2}} \sqrt{\frac{x}{\rho}}\right) \mathrm{d} x \\
& =\frac{1}{\log (2) \sigma^{2} \sqrt{\rho}} \mathrm{G}_{1,3}^{3,1}\left(\frac{1}{\sigma^{4} \rho} \mid-\frac{1}{2},-\frac{1}{2}, \frac{1}{2}\right)
\end{aligned}
$$

where $\mathrm{G}_{p, q}^{m, n}(\cdot)$ is the Meijer G function [28]. Here we have represented the Bessel function in terms of Meijer G function and subsequently use [28, Eq. 7.811.5]. 


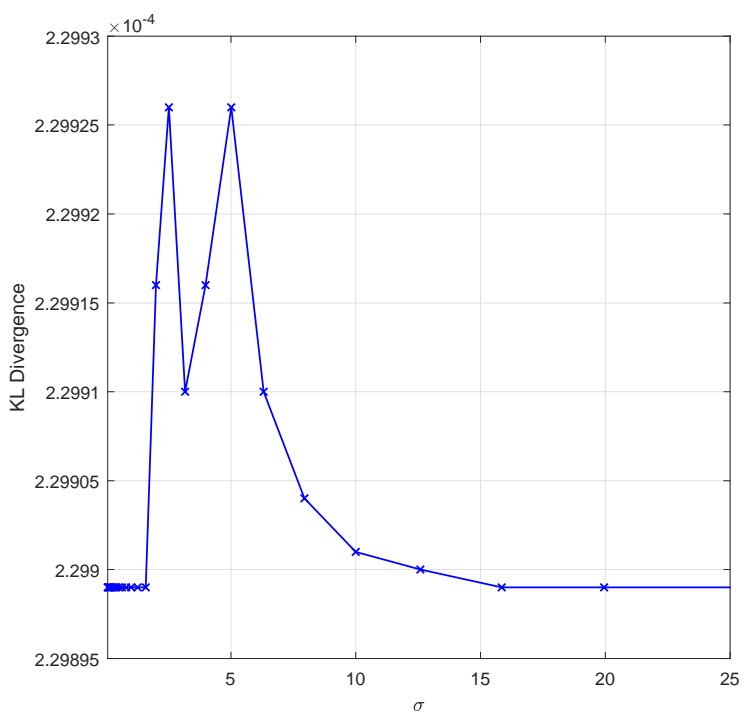

(a) The KL divergence vs $\sigma$.

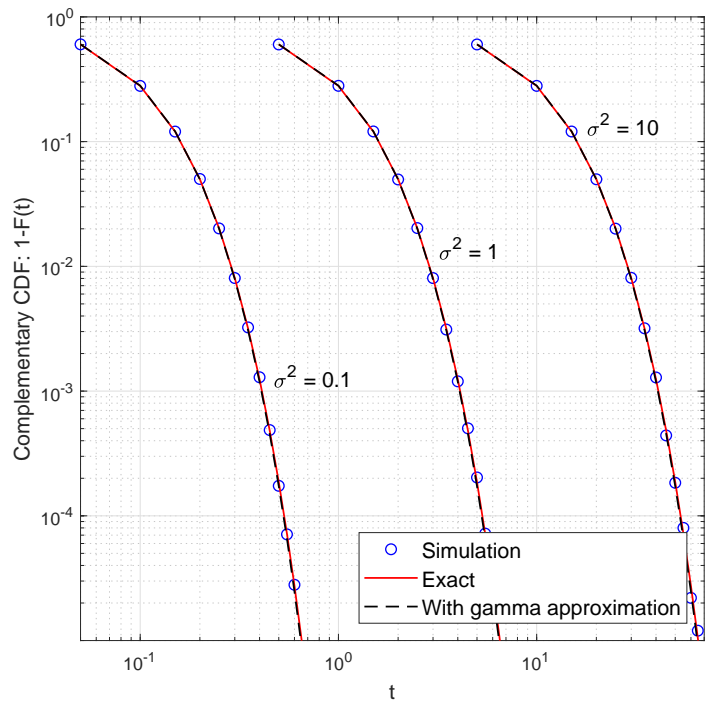

(b) The complementary CDF (CCDF) of $\zeta_{\ell}$.

Fig. 3: The comparison between the exact and approximation.

2) When $L \geq 2$ : Thus, with the aid of (21) and (23), the average spectral efficiency can be evaluated as

$$
\begin{aligned}
\mathrm{R}_{\mathrm{L} \geq 2}(\rho) \approx & \frac{1}{\log (2) \Gamma(L k)} \int_{0}^{\infty} \frac{1}{(1+x)} \Gamma\left(L k, \frac{1}{\theta} \sqrt{\frac{x}{\rho}}\right) \mathrm{d} x \\
= & \frac{1}{\log (2)}\left[2 \log (\theta)+\log (\rho)+2 \psi^{(0)}(L k)\right. \\
& +\frac{{ }_{2} \mathrm{~F}_{3}\left(1,1 ; 2, \frac{3}{2}-\frac{L k}{2}, 2-\frac{L k}{2} ;-\frac{1}{4 \theta^{2} \rho}\right)}{\theta^{2} \rho\left(k^{2} L^{2}-3 L k+2\right)} \\
& +\frac{\pi \rho^{-\frac{1}{2}(L k)}}{\theta^{L k} \Gamma(L k)}\left(\frac{{ }_{1} \mathrm{~F}_{2}\left(\frac{L k}{2} ; \frac{1}{2}, \frac{L k}{2}+1 ;-\frac{1}{4 \theta^{2} \rho}\right)}{L k\left(\csc \left(\frac{\pi L k}{2}\right)\right)^{-1}}\right. \\
& \left.\left.-\frac{{ }_{1} \mathrm{~F}_{2}\left(\frac{L k}{2}+\frac{1}{2} ; \frac{3}{2}, \frac{L k}{2}+\frac{3}{2} ;-\frac{1}{4 \theta^{2} \rho}\right)}{\sqrt{\rho} \theta(1+L k)\left(\sec \left(\frac{\pi L k}{2}\right)\right)^{-1}}\right)\right]
\end{aligned}
$$

where ${ }_{p} \mathrm{~F}_{q}(\cdot ; \cdot ; \cdot)$ is the generalized hypergeometric functions [28] and $\psi^{(0)}(z)$ is the logarithmic Gamma function [28]. Here we have represented the Gamma function in terms of hypergeometric functions and subsequently use respective integration in [28, Sec. 7.5].

\section{Asymptotic Analysis}

1) High SINR: The behavior of the outage probability at high SINR regime is given in the following theorem.

Theorem 1. For high SINR, i.e., $\rho \gg 1$, the user outage probability of $L$ elements RIS-assisted two-way networks decreases with the rate of $(\log (\rho) / \rho)^{L}$ over Rayleigh fading channels.

Proof: See Appendix A.
However, with a traditional multiple-relay network, we observe $(1 / \rho)^{L}$ rate. Since the end-to-end effective channel behaves as a product of two Rayleigh channels, we observe $(\log (\rho) / \rho)^{L}$ rate with a RIS network. This is one of the important observations found through this analysis, and, to the best of our knowledge, this behavior has not been captured in any of the previously published work.

The behavior of the average throughout at high SINR regime is given in the following theorem.

Theorem 2. For high SINR, i.e., $\rho \gg 1$, the user average spectral efficiency of $L$ elements RIS-assisted two-way networks increases with the rate of $\log (\rho)$ over Rayleigh fading channels.

\section{Proof: See Appendix B}

Since the residual self-interference may also be a function of the transmit power, it is worth discussing the behavior of the outage probability and average spectral efficiency when the transmit power is relatively larger than the noise and loop interference powers. For brevity, without loss of generality, we assume $P_{1}=P_{2}=P$. The following lemmas provide important asymptotic results.

Lemma 2. When the transmit power is relatively larger than the noise and loop interference, i.e., $P \gg \omega, \sigma_{w}^{2}$, the outage probabilities for $L=1$ and $L \geq 2$ vary, respectively, as

$$
P_{\mathrm{out} \mid \mathrm{L}=1}^{\infty} \rightarrow \begin{cases}\frac{\gamma_{\mathrm{th}}\left(\omega+\sigma_{w}^{2}\right)}{\sigma^{4}} \frac{\log (P)}{P} & \text { for } \sigma_{i}^{2}=\omega \\ P_{\mathrm{out} \mid \mathrm{L}=1}\left(\rho=\frac{1}{\omega}\right) & \text { for } \sigma_{i}^{2}=\omega P\end{cases}
$$

and

$$
P_{\mathrm{out} \mid \mathrm{L} \geq 2}^{\infty} \rightarrow \begin{cases}\mathcal{G}\left(L, \gamma_{\mathrm{th}}, \omega, \sigma\right)\left(\frac{\log (P)}{P}\right)^{L} & \text { for } \sigma_{i}^{2}=\omega \\ P_{\mathrm{out} \mid \mathrm{L} \geq 2}\left(\rho=\frac{1}{\omega}\right) & \text { for } \sigma_{i}^{2}=\omega P\end{cases}
$$


where $\mathcal{G}\left(L, \gamma_{\mathrm{th}}, \omega, \sigma\right)$ is the array gain. While the outage probability decreases with the rate $\left(\frac{\log (P)}{P}\right)^{L}$ for $\sigma_{i}^{2}=\omega$, there is an outage floor for $\sigma_{i}^{2}=\omega P$.

Proof: In particular, we consider the following two extreme cases:

1) When $\sigma_{i}^{2}=\omega$, where the interference is independent of the transmit power, we have $\rho=P /\left(\omega+\sigma_{w}^{2}\right) \stackrel{P \gg \omega, \sigma_{w}^{2}}{\longrightarrow}$ $\rho \propto P$. Therefore, results can easily be deduced from Theorem 2. Please note that we derive $L \geq 2$ case with upper and lower bounds, it is still unclear the precise expression for the array gain $\mathcal{G}\left(L, \gamma_{\mathrm{th}}, \omega, \sigma\right)$. We thus leave is as a future work.

2) When $\sigma_{i}^{2}=\omega P$, where the interference is proportional to the transmit power, we have $\rho=P /(\omega P+$ $\left.\sigma_{w}^{2}\right) \stackrel{P \gg \omega, \sigma_{w}^{2}}{\longrightarrow} \rho \propto 1 / \omega$. This means that the loopinterference variance dominates the outage probability, and respective asymptotic results can be obtained from (16) and (22) replacing $\rho$ by $1 / \omega$.

This completes the proof.

When $\sigma_{i}^{2}=\omega P^{\nu}$ where $\nu \in(0,1)$, it is not trivial to expand the outage probability expressions with respect to $P$ for rational $\nu$, we omit this case. However, the performance of this case is in between $\nu=0$ and $\nu=1$ cases.

Lemma 3. For $P \gg \omega, \sigma_{w}^{2}$, the average spectral efficiency for $L=1$ and $L \geq 2$ vary, respectively, as

$$
\mathrm{R}_{L=1}^{\infty} \rightarrow \begin{cases}\frac{\log (P)-\log \left(\frac{\omega+\sigma_{w}^{2}}{\sigma^{4}}\right)-2 \epsilon}{\log (2)} & \text { for } \sigma_{i}^{2}=\omega \\ \mathrm{R}_{L=1}\left(\rho=\frac{1}{\omega}\right) & \text { for } \sigma_{i}^{2}=\omega P\end{cases}
$$

and

$$
\mathrm{R}_{L \geq 2}^{\infty} \rightarrow \begin{cases}\frac{\log (P)+2 \psi^{(0)}(L k)-\log \left(\frac{\sigma_{w}^{2}+\omega}{\theta^{2}}\right)}{\log (2)} ; & \sigma_{i}^{2}=\omega \\ \mathrm{R}_{L \geq 2}\left(\rho=\frac{1}{\omega}\right) ; & \sigma_{i}^{2}=\omega P\end{cases}
$$

While the average spectral efficiency increases with the rate $\log (P)$ for $\sigma_{i}^{2}=\omega$, there is a spectral efficiency floor for $\sigma_{i}^{2}=\omega P$.

Proof: Since the proof follows the similar steps as Lemma 2, we omit the details.

Lemma 3 also reveals that the average spectral efficiency increases with $L$ because $\psi^{(0)}(x)$ is an increasing function. Further, when number of elements increases from $L_{1}$ to $L_{2}(\geq$ $L_{1}$ ), we have $\Delta \mathrm{R}$ spectral efficiency improvements for any given $P$ where

$$
\Delta \mathrm{R}=\frac{2\left(\psi^{(0)}\left(L_{2} k\right)-\psi^{(0)}\left(L_{1} k\right)\right)}{\log (2)}[\mathrm{bits} / \mathrm{sec} / \mathrm{Hz}] .
$$

On the other hand, we can also save $\Delta P$ power for any given $\mathrm{R}$ where

$$
\Delta P=20 \log _{10}(\mathrm{e})\left(\psi^{(0)}\left(L_{2} k\right)-\psi^{(0)}\left(L_{1} k\right)\right)[\mathrm{dBm}]
$$

Based on the behavior of $\psi^{(0)}(x)$ function, the rates of $\Delta \mathrm{R}$ increment and $\Delta P$ saving decrease with $L$. Thus, use of a very large number of elements at the RIS may not be effective compared to the required overhead cost for large number of channel estimations and phase adjustments.
2) For Large L (or LIS): For a sufficiently large number $L$, according to the central limit theorem (CLT), the RV $\zeta=$ $\sum_{\ell=1}^{L} \zeta_{\ell}$ converges to a Gaussian random variable with $\mu=$ $L \pi \sigma^{2} / 4$ mean and $\eta=L\left(16-\pi^{2}\right) \sigma^{4} / 16$ variance which has the CDF expression

$$
F_{\zeta}(t)=\frac{1}{2}\left(1+\operatorname{erf}\left[\frac{t-\mu}{\sqrt{2 \eta}}\right]\right) ; \quad t \in(-\infty,+\infty)
$$

where erf [.] is the Gauss error function [28]. Since the CDF of $\gamma=\rho \zeta^{2}$ is given as $F_{\gamma}(t)=F_{\zeta}(\sqrt{t / \rho})-F_{\zeta}(-\sqrt{t / \rho})$, the outage probability can be evaluated as

$$
\begin{aligned}
P_{\text {out } \mid \mathrm{L} \gg 1} & \approx \frac{1}{2}\left(\operatorname{erf}\left[\frac{\sqrt{\frac{\gamma_{\text {th }}}{\rho}}-\mu}{\sqrt{2 \eta}}\right]+\operatorname{erf}\left[\frac{\sqrt{\frac{\gamma_{\text {th }}}{\rho}}+\mu}{\sqrt{2 \eta}}\right]\right) \\
& =1-Q_{\frac{1}{2}}\left(\frac{\mu}{\sqrt{\eta}}, \sqrt{\frac{\gamma_{\text {th }}}{\eta \rho}}\right)
\end{aligned}
$$

where $\mathrm{Q}_{m}(\cdot, \cdot)$ is the Marcum's Q-function and the second equality follows from the results in [31].

However, this CLT approximation may not be helpful to derive the average spectral efficiency in closed-form or with inbuilt special functions, which may also be a disadvantage of this approach.

\section{E. Discussion on Scheme 2}

For Scheme 2, the maximum instantaneous SNR of each user is

$$
\gamma=\frac{P}{\sigma_{w}^{2}}\left(\sum_{\ell=1}^{L} \zeta_{\ell}\right)^{2}
$$

where $P$ is the transmit power. The corresponding optimal phases are $\phi_{\ell}^{\star}=\varphi_{\ell}+\psi_{\ell}$ for both users with reciprocal channels, $\phi_{\ell}^{\star}=\varphi_{\mathrm{r}, \ell}+\psi_{\mathrm{t}, \ell}$ for $U_{1}$ with non-reciprocal channels, and $\phi_{\ell}^{\star}=\varphi_{\mathrm{t}, \ell}+\psi_{\mathrm{r}, \ell}$ for $U_{2}$ with non-reciprocal channels. It is important to note that there is no loop interference, i.e., $\sigma_{i}^{2}=0$ in (4), (5), (7) or (8). Therefore, the SNR of Scheme 2 is always larger than the SINR of Scheme 1, and achieves lower outage probability which can easily be deduced from (16) and (22) replacing $\rho$ as $\rho=P / \sigma_{w}^{2}$. From Theorem 2, we can conclude that the user outage probability decreases with the rate of $(\log (\rho) / \rho)^{L}$ over Rayleigh fading channels.

Since only one user communicates in a given frequency or time resource block, we have factor $1 / 2$ for the average spectral efficiency in (23). It can then be derived by multiplying factor $1 / 2$ and replacing $\rho$ as $\rho=P / \sigma_{w}^{2}$ of (24) and (25).

With respect to the average spectral efficiency, we now discuss which transmission scheme is better for a given transmit power $P$. Since the direct comparison by using the spectral efficiency expressions in (24) and (25) for Scheme 1 and Scheme 2 does not yield any tractable analytical expressions for $P$, we compare their asymptotic expressions where the corresponding spectral efficiency expressions for Scheme 2 can be given with the aid of (28) and (29) as

$$
\mathrm{R}_{\mathrm{one}}^{\infty} \rightarrow\left\{\begin{array}{ll}
\frac{\log (P)-\log \left(\frac{\sigma_{w}^{2}}{\sigma^{4}}\right)-2 \epsilon}{2 \log (2)} ; & \text { for } L=1 \\
\frac{\log (P)+2 \psi^{(0)}(L k)-\log \left(\frac{\sigma_{w}^{2}}{\theta^{2}}\right)}{2 \log (2)} ; & \text { for } L \geq 2
\end{array} .\right.
$$


Now we seek the condition for which Scheme 1 outperforms Scheme 2.

Lemma 4. The transmit power boundary where Scheme 1 outperforms Scheme 2 can be approximately given

- for $\sigma_{i}^{2}=\omega$ as

$$
P> \begin{cases}\left(\frac{\omega+\sigma_{w}^{2}}{\sigma_{w} \sigma^{2}}\right)^{2} \mathrm{e}^{2 \epsilon} ; & \text { for } L=1 \\ \left(\frac{\omega+\sigma_{w}^{2}}{\sigma_{w} \theta}\right)^{2} \mathrm{e}^{-2 \psi^{(0)}(L k)} ; & \text { for } L \geq 2 .\end{cases}
$$

and

- for $\sigma_{i}^{2}=\omega P$ as

$$
P< \begin{cases}\mathrm{e}^{2 \log (2) \mathrm{R}_{L=1}\left(\frac{1}{\omega}\right)+\log \left(\frac{\sigma_{w}^{2}}{\sigma^{4}}\right)+2 \epsilon} ; & L=1 \\ \mathrm{e}^{2 \log (2) \mathrm{R}_{L \geq 2}\left(\frac{1}{\omega}\right)+\log \left(\frac{\sigma_{w}^{2}}{\theta^{2}}\right)-2 \psi^{(0)}(L k)} & ; L \geq 2\end{cases}
$$

Proof: We can derive these with direct comparisons $\mathrm{R}_{\mathrm{one}}^{\infty}<\mathrm{R}_{\mathrm{L}=1}^{\infty}$ and $\mathrm{R}_{\mathrm{one}}^{\infty}<\mathrm{R}_{\mathrm{L} \geq 2}^{\infty}$ by using (28), (29) and (36).

\section{Network With Non-Reciprocal CHANNELS}

In this case, the SINR at $U_{1}$ in (7) and the SINR at $U_{2}$ in (8) can be alternatively given as

$$
\begin{aligned}
& \gamma_{1}=\rho_{1}\left|\sum_{\ell=1}^{L} c_{1, \ell} \mathrm{e}^{j\left(\phi_{\ell}-\varphi_{\mathrm{r}, \ell}-\psi_{\mathrm{t}, \ell}\right)}\right|^{2} \text { and } \\
& \gamma_{2}=\rho_{2}\left|\sum_{\ell=1}^{L} c_{2, \ell} \mathrm{e}^{j\left(\phi_{\ell}-\psi_{\mathrm{r}, \ell}-\varphi_{\mathrm{t}, \ell}\right)}\right|^{2}
\end{aligned}
$$

where $\rho_{1}=P_{2} /\left(\sigma_{i_{1}}^{2}+\sigma_{w_{1}}^{2}\right), c_{1, \ell}=\alpha_{\mathrm{r}, \ell} \beta_{\mathrm{t}, \ell}, \rho_{2}=P_{1} /\left(\sigma_{i_{2}}^{2}+\right.$ $\left.\sigma_{w_{2}}^{2}\right)$ and $c_{2, \ell}=\beta_{\mathrm{r}, \ell} \alpha_{\mathrm{t}, \ell}$.

By looking at the structures of $\gamma_{1}$ and $\gamma_{2}$, finding the optimal $\phi_{\ell}$, which maximizes the instantaneous SINR of each user, is not straightforward as in the case with reciprocal channels. This stems from the fact that the optimal $\phi_{\ell}$ in this case depends on phases of all channels $\varphi_{\mathrm{r}, \ell}, \psi_{\mathrm{t}, \ell}, \psi_{\mathrm{r}, \ell}$ and $\varphi_{\mathrm{t}, \ell}$, and also the SINR $\gamma_{1}$ is a function of $\varphi_{\mathrm{r}, \ell}, \psi_{\mathrm{t}, \ell}$, and the SINR $\gamma_{2}$ is a function of $\psi_{\mathrm{r}, \ell}, \varphi_{\mathrm{t}, \ell}$. In this section, the optimization problem for maximizing the minimum user SINR, i.e., $\min \left(\gamma_{1}, \gamma_{2}\right)$, is to be formulated by optimizing the phase of the $\ell$ th element of the RIS, i.e., $\phi_{\ell}, \forall l \in \mathcal{L}$. We consider $L=1$ and $L \geq 2$ cases separately.

\section{A. For $L=1$}

In this case, we have

$$
\begin{aligned}
& \gamma_{1}=\rho_{1}\left|c_{1,1} \mathrm{e}^{j\left(\phi_{1}-\varphi_{\mathrm{r}, 1}-\psi_{\mathrm{t}, 1}\right)}\right|^{2}=\rho_{1} c_{1,1}^{2} \text { and } \\
& \gamma_{2}=\rho_{2}\left|c_{2,1} \mathrm{e}^{j\left(\phi_{1}-\psi_{\mathrm{r}, 1}-\varphi_{\mathrm{t}, 1}\right)}\right|^{2}=\rho_{2} c_{2,1}^{2} .
\end{aligned}
$$

Due to the fact that $\left|\mathrm{e}^{j \theta}\right|=1$, the phase of each element can be any arbitrary angle which can be assigned randomly. Further, the outage probability and average spectral efficiency are the same as (16) and (24).

\section{B. For $L \geq 2$ : Problem Formulation}

For general $L$, define $\phi=\left(\phi_{1}, \cdots, \phi_{\ell}, \cdots, \phi_{L}\right)^{T}$, and the optimization problem that maximizes $\min \left(\gamma_{1}, \gamma_{2}\right)$ can be written as the following form

Problem 1.

$$
\begin{array}{ll}
\max _{\phi} & \min \left(\gamma_{1}, \gamma_{2}\right) \\
\text { s.t. } & \phi_{l} \in[0,2 \pi), \forall l \in \mathcal{L} .
\end{array}
$$

which is equivalent with the following optimization problem

\section{Problem 2.}

$$
\begin{array}{ll}
\max _{\phi} & t \\
\text { s.t. } & \gamma_{1} \geq t, \\
& \gamma_{2} \geq t, \\
& \phi_{l} \in[0,2 \pi), \forall l \in \mathcal{L} .
\end{array}
$$

Problem 2 is hard to solve directly, since both $\gamma_{1}$ and $\gamma_{2}$ are non-convex functions with $\boldsymbol{\Phi}$.

\section{For $L \geq 2$ : Solution}

To get the optimal solution of Problem 2, we will make the following transformations.

In the first step, by resorting to (39), we can re-write $\gamma_{1}$ and $\gamma_{2}$ as in (42) and (43), respectively, which are given on the top of this page.

Define following $2 L$-dimensional vectors:

$$
\begin{aligned}
\alpha \triangleq\left(\cos \left(\phi_{1}\right), \sin \left(\phi_{1}\right),\right. \\
\left.\quad \ldots, \cos \left(\phi_{l}\right), \sin \left(\phi_{l}\right), \ldots, \cos \left(\phi_{L}\right), \sin \left(\phi_{L}\right)\right)^{T}
\end{aligned}
$$

$\boldsymbol{c}_{1} \triangleq \sqrt{\rho_{1}}\left(c_{1,1} \cos \left(\varphi_{\mathrm{r}, 1}+\psi_{\mathrm{t}, 1}\right), c_{1,1} \sin \left(\varphi_{\mathrm{r}, 1}+\psi_{\mathrm{t}, 1}\right)\right.$, $\left.\ldots, c_{1, L} \cos \left(\varphi_{\mathrm{r}, L}+\psi_{\mathrm{t}, L}\right), c_{1, L} \sin \left(\varphi_{\mathrm{r}, L}+\psi_{\mathrm{t}, L}\right)\right)^{T}$,

$\boldsymbol{d}_{1} \triangleq \sqrt{\rho_{1}}\left(-c_{1,1} \sin \left(\varphi_{\mathrm{r}, 1}+\psi_{\mathrm{t}, 1}\right), c_{1,1} \cos \left(\varphi_{\mathrm{r}, 1}+\psi_{\mathrm{t}, 1}\right)\right.$,

$$
\left.\ldots,-c_{1, L} \sin \left(\varphi_{\mathrm{r}, L}+\psi_{\mathrm{t}, L}\right), c_{1, L} \cos \left(\varphi_{\mathrm{r}, L}+\psi_{\mathrm{t}, L}\right)\right)^{T},
$$

$\boldsymbol{c}_{2} \triangleq \sqrt{\rho_{2}}\left(c_{2,1} \cos \left(\psi_{\mathrm{r}, 1}+\varphi_{\mathrm{t}, 1}\right), c_{2,1} \sin \left(\psi_{\mathrm{r}, 1}+\varphi_{\mathrm{t}, 1}\right)\right.$,

$$
\left.\ldots, c_{2, L} \cos \left(\psi_{\mathrm{r}, L}+\varphi_{\mathrm{t}, L}\right), c_{2, L} \sin \left(\psi_{\mathrm{r}, L}+\varphi_{\mathrm{t}, L}\right)\right)^{T},
$$

$$
\begin{gathered}
\boldsymbol{d}_{2} \triangleq \sqrt{\rho_{2}}\left(-c_{2,1} \sin \left(\psi_{\mathrm{r}, 1}+\varphi_{\mathrm{t}, 1}\right), c_{2,1} \cos \left(\psi_{\mathrm{r}, 1}+\varphi_{\mathrm{t}, 1}\right)\right. \\
\left.\ldots,-c_{2, L} \sin \left(\psi_{\mathrm{r}, L}+\varphi_{\mathrm{t}, L}\right), c_{2, L} \cos \left(\psi_{\mathrm{r}, L}+\varphi_{\mathrm{t}, L}\right)\right)^{T}
\end{gathered}
$$

Then $\gamma_{1}$ and $\gamma_{2}$ can be written as the quadratic form of $\boldsymbol{\alpha}$ as follows

$$
\gamma_{1}=\boldsymbol{c}_{1}^{T} \boldsymbol{\alpha} \boldsymbol{\alpha}^{T} \boldsymbol{c}_{1}+\boldsymbol{d}_{1}^{T} \boldsymbol{\alpha} \boldsymbol{\alpha}^{T} \boldsymbol{d}_{1}
$$

and

$$
\gamma_{2}=\boldsymbol{c}_{2}^{T} \boldsymbol{\alpha} \boldsymbol{\alpha}^{T} \boldsymbol{c}_{2}+\boldsymbol{d}_{2}^{T} \boldsymbol{\alpha} \boldsymbol{\alpha}^{T} \boldsymbol{d}_{2}
$$




$$
\begin{aligned}
\gamma_{1}= & \rho_{1}\left|\sum_{\ell=1}^{L} c_{1, \ell}\left(\cos \left(\phi_{\ell}-\varphi_{\mathrm{r}, \ell}-\psi_{\mathrm{t}, \ell}\right)+i \sin \left(\phi_{\ell}-\varphi_{\mathrm{r}, \ell}-\psi_{\mathrm{t}, \ell}\right)\right)\right|^{2} \\
= & \rho_{1} \mid \sum_{\ell=1}^{L} c_{1, \ell}\left(\cos \left(\phi_{\ell}\right) \cos \left(\varphi_{\mathrm{r}, \ell}+\psi_{\mathrm{t}, \ell}\right)+\sin \left(\phi_{\ell}\right) \sin \left(\varphi_{\mathrm{r}, \ell}+\psi_{\mathrm{t}, \ell}\right)\right. \\
& \left.+i\left(\sin \left(\phi_{\ell}\right) \cos \left(\varphi_{\mathrm{r}, \ell}+\psi_{\mathrm{t}, \ell}\right)-\cos \left(\phi_{\ell}\right) \sin \left(\varphi_{\mathrm{r}, \ell}+\psi_{\mathrm{t}, \ell}\right)\right)\right)\left.\right|^{2} \\
= & \rho_{1}\left(\sum_{\ell=1}^{L} c_{1, \ell}\left(\cos \left(\phi_{\ell}\right) \cos \left(\varphi_{\mathrm{r}, \ell}+\psi_{\mathrm{t}, \ell}\right)+\sin \left(\phi_{\ell}\right) \sin \left(\varphi_{\mathrm{r}, \ell}+\psi_{\mathrm{t}, \ell}\right)\right)\right)^{2} \\
& +\rho_{1}\left(\sum_{\ell=1}^{L} c_{1, \ell}\left(\sin \left(\phi_{\ell}\right) \cos \left(\varphi_{\mathrm{r}, \ell}+\psi_{\mathrm{t}, \ell}\right)-\cos \left(\phi_{\ell}\right) \sin \left(\varphi_{\mathrm{r}, \ell}+\psi_{\mathrm{t}, \ell}\right)\right)\right)^{2}
\end{aligned}
$$

and

$$
\begin{aligned}
\gamma_{2}= & \rho_{2}\left|\sum_{\ell=1}^{L} c_{2, \ell}\left(\cos \left(\phi_{\ell}-\psi_{\mathrm{r}, \ell}-\varphi_{\mathrm{t}, \ell}\right)+i \sin \left(\phi_{\ell}-\psi_{\mathrm{r}, \ell}-\varphi_{\mathrm{t}, \ell}\right)\right)\right|^{2} \\
= & \rho_{2} \mid \sum_{\ell=1}^{L} c_{2, \ell}\left(\cos \left(\phi_{\ell}\right) \cos \left(\psi_{\mathrm{r}, \ell}+\varphi_{\mathrm{t}, \ell}\right)+\sin \left(\phi_{\ell}\right) \sin \left(\psi_{\mathrm{r}, \ell}+\varphi_{\mathrm{t}, \ell}\right)\right. \\
& \left.+i\left(\sin \left(\phi_{\ell}\right) \cos \left(\psi_{\mathrm{r}, \ell}+\varphi_{\mathrm{t}, \ell}\right)-\cos \left(\phi_{\ell}\right) \sin \left(\psi_{\mathrm{r}, \ell}+\varphi_{\mathrm{t}, \ell}\right)\right)\right)\left.\right|^{2} \\
= & \rho_{2}\left(\sum_{\ell=1}^{L} c_{2, \ell}\left(\cos \left(\phi_{\ell}\right) \cos \left(\psi_{\mathrm{r}, \ell}+\varphi_{\mathrm{t}, \ell}\right)+\sin \left(\phi_{\ell}\right) \sin \left(\psi_{\mathrm{r}, \ell}+\varphi_{\mathrm{t}, \ell}\right)\right)\right)^{2} \\
& +\rho_{2}\left(\sum_{\ell=1}^{L} c_{2, \ell}\left(\sin \left(\phi_{\ell}\right) \cos \left(\psi_{\mathrm{r}, \ell}+\varphi_{\mathrm{t}, \ell}\right)-\cos \left(\phi_{\ell}\right) \sin \left(\psi_{\mathrm{r}, \ell}+\varphi_{\mathrm{t}, \ell}\right)\right)\right)^{2}
\end{aligned}
$$

Define $\boldsymbol{A}=\boldsymbol{\alpha} \boldsymbol{\alpha}^{T}, \boldsymbol{C}_{1}=\boldsymbol{c}_{1} \boldsymbol{c}_{1}^{T}, \boldsymbol{D}_{1}=\boldsymbol{d}_{1} \boldsymbol{d}_{1}^{T}, \boldsymbol{F}_{1}=\boldsymbol{C}_{1}+\boldsymbol{D}_{1}$, $\boldsymbol{C}_{2}=\boldsymbol{c}_{2} \boldsymbol{c}_{2}^{T}, \boldsymbol{D}_{2}=\boldsymbol{d}_{2} \boldsymbol{d}_{2}^{T}$, and $\boldsymbol{F}_{2}=\boldsymbol{C}_{2}+\boldsymbol{D}_{2}$. It can be easily checked that the matrixes $A, C_{1}, C_{2}, D_{1}, D_{2}, F_{1}$, and $\boldsymbol{F}_{2}$ are all semi-definite positive matrixes. With the above denotations, $\gamma_{1}$ and $\gamma_{2}$ can be further written as

$$
\begin{gathered}
\gamma_{1}=\operatorname{Tr}\left(\left(\boldsymbol{C}_{1}+\boldsymbol{D}_{1}\right) \boldsymbol{A}\right)=\operatorname{Tr}\left(\boldsymbol{F}_{1} \boldsymbol{A}\right)=\boldsymbol{F}_{1} \bullet \boldsymbol{A} \\
\gamma_{2}=\operatorname{Tr}\left(\left(\boldsymbol{C}_{2}+\boldsymbol{D}_{2}\right) \boldsymbol{A}\right)=\operatorname{Tr}\left(\boldsymbol{F}_{2} \boldsymbol{A}\right)=\boldsymbol{F}_{2} \bullet \boldsymbol{A} .
\end{gathered}
$$

For the matrix $\boldsymbol{A}$, since it is composed of $\sin \left(\phi_{l}\right)$ and $\cos \left(\phi_{l}\right)$, and $\sin \left(\phi_{l}\right)^{2}+\cos \left(\phi_{l}\right)^{2}=1$ for $l \in \mathcal{L}, \boldsymbol{A}$ has to satisfy the following constraint

$$
\boldsymbol{I}_{l} \bullet \boldsymbol{A}=1, \forall l \in \mathcal{L}
$$

where $\boldsymbol{I}_{l}$ is the square matrix with $(2 l-1)$ th and $2 l$ th diagonal element being 1 and all the other elements being 0 . In addition, the rank of $\boldsymbol{A}$ should be 1. Collecting the aforementioned constraints on $\boldsymbol{A}$, Problem 2 can be reformulated as the following optimization problem

\section{Problem 3.}

$$
\begin{array}{cc}
\max _{\boldsymbol{A}} & t \\
\text { s.t. } & \boldsymbol{F}_{1} \bullet \boldsymbol{A} \geq t, \\
& \boldsymbol{F}_{2} \bullet \boldsymbol{A} \geq t, \\
& \boldsymbol{I}_{l} \bullet \boldsymbol{A}=1, \forall l \in \mathcal{L}, \\
& \operatorname{Rank}(\boldsymbol{A})=1, \\
\boldsymbol{A} \succeq 0
\end{array}
$$

where $\boldsymbol{A} \succeq 0$ indicates that the matrix $\boldsymbol{A}$ is semi-definite matrix.

Problem 3 is also a non-convex optimization problem due to the constraint (49d). We relax Problem 3 by dropping the constraint (49d), then Problem 3 turns to be the following optimization problem

\section{Problem 4.}

$$
\begin{array}{ll}
\max _{\boldsymbol{A}} & t \\
\text { s.t. } & \boldsymbol{F}_{1} \bullet \boldsymbol{A} \geq t, \\
& \boldsymbol{F}_{2} \bullet \boldsymbol{A} \geq t, \\
& \boldsymbol{I}_{l} \bullet \boldsymbol{A}=1, \forall l \in \mathcal{L}, \\
& \boldsymbol{A} \succeq 0
\end{array}
$$

For given $t$, Problem 4 is a SDP feasibility problem, which can be solved with the help of CVX toolbox [32]. Note that the complexity for solving Problem 4 with $t$ given can be at the scale of $O(\sqrt{2 L})$ according to [33]. Then we need to find the maximal achievable $t$, which can be found by resorting to bisection-search method. Denote the initial two boundary value of $t$ are $t_{L}$ and $t_{U}$ respectively, where $t_{L}$ makes Problem 4 feasible and $t_{U}$ makes Problem 4 infeasible. Hence the number of iterations to achieve $\varepsilon$-tolerance, which can guarantee the difference between the searched $t$ and the maximal $t$ enabling Problem 4 to be feasible lies between $\varepsilon$, is $O\left(\log \left(\frac{\left(t_{U}-t_{L}\right)}{\varepsilon}\right)\right) \sqrt{2 L}$. Note that this complexity is polynomial with $L$. To this end, the optimal solution of Problem 4 has been found and the associated complexity has been characterized.

In the last step, we need to find a rank-1 solution of Problem 4. One broadly used method is "Gaussian randomization procedure" in [34]. By following the idea of Gaussian random procedure, the rank-1 solution can be found in Algorithm 1. 


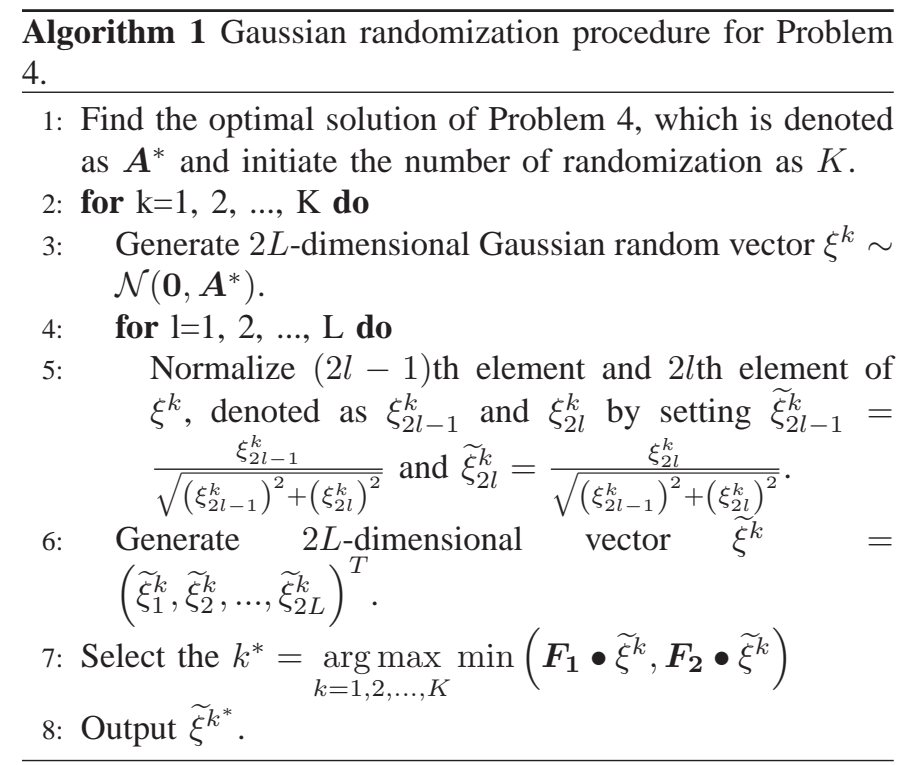

Remark: In the real application, when $K$ is larger, better solution for Problem 4 can be achieved, which, however, will lead to higher computation complexity. A balanced selection of $K$ is required.

\section{Simulation Results}

In this section, we investigate the performance of the RISaided two-way networks. We set channel variance $\sigma^{2}=1$. Since the thermal noise floor for $1 \mathrm{~Hz}$ bandwidth at room temperature can even be $-174 \mathrm{dBm}$, we use $-70 \mathrm{dBm}$ to represent a more noisy scenario. All presented illustrations include average results over $10^{6}$ and $10^{3}$ independent channel realizations for the outage probability and the average spectral efficiency calculations, respectively.

\section{A. For $L=1$ with Reciprocal Channels}

Fig. 4 shows the outage probability and average spectral efficiency vs $P$ for $L=1$. Several observations are gained: i) Our analytical results in (16) and (24) exactly match with the simulation results, which confirms the accuracy of our analysis; ii) For different loop interference $\sigma_{i}^{2}-\omega P^{\nu}$, we notice that the outage decreases at a rate of $\log (P) / P$ and the spectral efficiency increases at a rate of $\log (P)$ when $\nu=0$, and both have floors when $\nu=1$ due to the transmit-power dependent interference. These have been analytically proved in (26) and (28). As we expect, when $\nu \in(0,1)$, e.g., $\nu=0.2$, the outage and spectral efficiency are in between $\nu=0$ and $\nu=1$ cases; iii) When $\omega$ reduces from $10^{-4}$ to $10^{-5}$, the outage and spectral efficiency improve around $9 \mathrm{~dB}$ and 3.32 [bits/sec/Hz], respectively, for each case; and iv) two-way communications with Scheme 2 outperforms Scheme 1 when $P<5 \mathrm{dBm}$ and $P<25 \mathrm{dBm}$ for $\omega=10^{-5}$ and $\omega=10^{-4}$, respectively, with $\nu=0$. For $\nu=1$, Scheme 2 outperforms Scheme 1 in the entire simulated region ${ }^{1}$. Therefore, it is important to keep the effect of loop interference independent of transmit power if two-way communications use Scheme 1.

\footnotetext{
${ }^{1}$ We do not show the outage probability of Scheme 2 because it always outperforms Scheme 1 as long as the loop interference is non-zero.
}

\section{B. For $L \geq 2$ with Reciprocal Channels}

For $L \geq 2$, Fig. 5 shows the outage probability vs $P$, when loop-interference is independent of transmit power $P$, i.e., $\sigma_{i}^{2}=\omega$. For a given $L$, the outage probability decreases with $[\log (P) / P]^{L}$ which confirms Lemma 2. Although the outage probability decreases with $L$, the diminishing rate also decreases, as discussed earlier with respect to (31). For example, when we increase $L$ from 2 to 4 , we can save power around $14 \mathrm{dBm}$ at $10^{-3}$ outage. However, for the same outage, we can only save power around $8 \mathrm{dBm}$ when we increase $L$ from 32 to 64. Interestingly, this figure confirms the accuracy of our gamma approximation. Moreover, it is more accurate than the CLT approximation even for $L=32$ or $L=64$.

For $L \geq 2$, Fig. 6 shows the spectral efficiency vs $P$, when loop-interference is independent of transmit power $P$, i.e., $\sigma_{i}^{2}=\omega$, and linearly dependent of transmit power $P$, i.e., $\sigma_{i}^{2}=\omega P$. For any $L$, as shown in Fig. 6a and (29), the average spectral efficiency increases in order of $\log (P)$ when $\sigma_{i}^{2}=\omega$, which confirms Lemma 3. According to the figure and (31), while transmit power reduces by around $19 \mathrm{dBm}$ when $L$ increases from 2 to 16 , we can only save $12 \mathrm{dBm}$ when $L$ increases from 16 to 64 . We also plot the spectral efficiency of Scheme 1 and Scheme 2 in Fig. 6a where Scheme 1 starts to outperform Scheme 2 when $P$ increases where transition happens at $P \approx 17.5,-1.8,-14.0 \mathrm{dBm}$ for $L=2,16,64$, respectively. This compliments Lemma 4 . Fig. $6 \mathrm{~b}$ is for $\sigma_{i}^{2}=\omega P$ where we have spectral efficiency floors because loop-interference enhances with transmit power in Scheme 1. Due to this reason, as shown in the figure, Scheme 2 outperforms Scheme 1 when $P$ increases.

Fig. 7 shows the transition boundary of transmit power $P$ where Scheme 1 outperforms Scheme 2 or vice versa. Based on the results in Lemma 4 and simulations, we plot $P$ vs $\omega$ for both cases $\sigma_{i}^{2}=\omega$ and $\sigma_{i}^{2}=\omega P$ in Fig. 7a and Fig. 7b, respectively. For $\sigma_{i}^{2}=\omega$, Scheme 1 outperforms at high $P$, and the $P$ decreases when $L$ increases for given $\omega$. We have opposite observation for the other case $\sigma_{i}^{2}=\omega P$. Moreover, when loop interference power is less than the noise power, i.e., $\omega<10^{-10}$, the noise power dominates, and we have power floor. For example, the power floor is around $-120 \mathrm{dBm}$ with $L=16$ for $\sigma_{i}^{2}=\omega$ case.

\section{For Non-reciprocal Channels}

Fig. 8a plots the spectral efficiency vs transmit power of both users for three types of phase adjustment techniques: 1) fairness Algorithm 1;2) phase is adjusted based on $U_{1}{ }^{2}$, i.e., $\phi_{\ell}=\varphi_{r, \ell}+\psi_{t, \ell}$; and 3) phase is randomly adjusted. $U_{1}$ and $U_{1}$ have the same spectral efficiency with Algorithm 1, which confirms the user fairness. When phase is adjusted based on $U_{1}, U_{1}$ has the best performance among all, and it has around $7.5 \%$ spectral efficiency improvement at $P=0 \mathrm{dBm}$ compared Algorithm 1. However, $U_{1}$ has the worst performance which is very similar the case of random phase adjustment where both users have similar performance but worst. The spectral

\footnotetext{
${ }^{2}$ We do not include results when the phase is adjusted based on $U_{1}$ because we achieve the similar behaviour.
} 


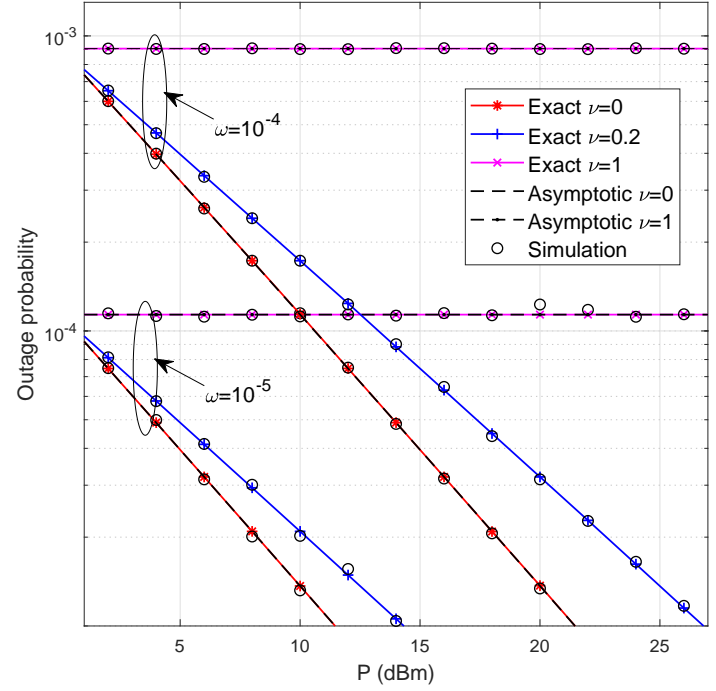

(a) The outage probability vs $P$.

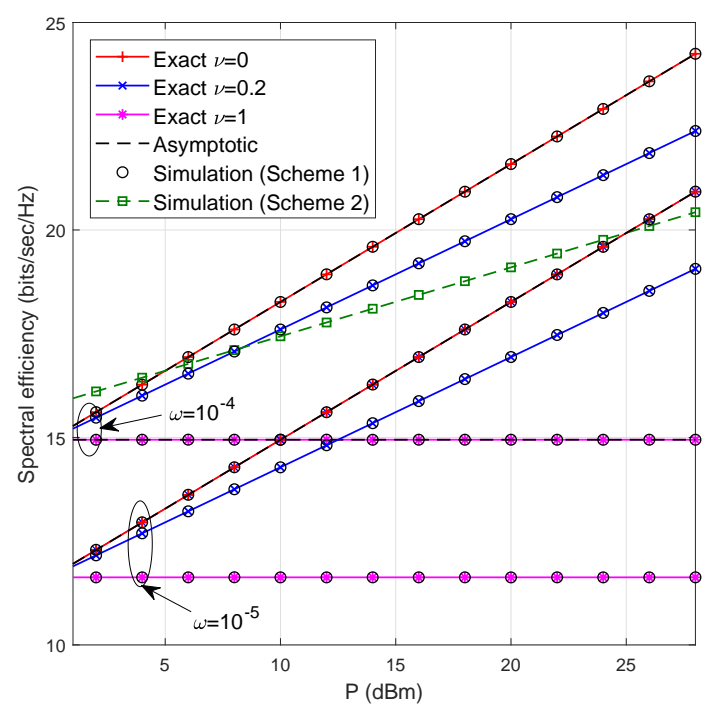

(b) The spectral efficiency vs $P$.

Fig. 4: The performance for reciprocal channels when $L=1$.

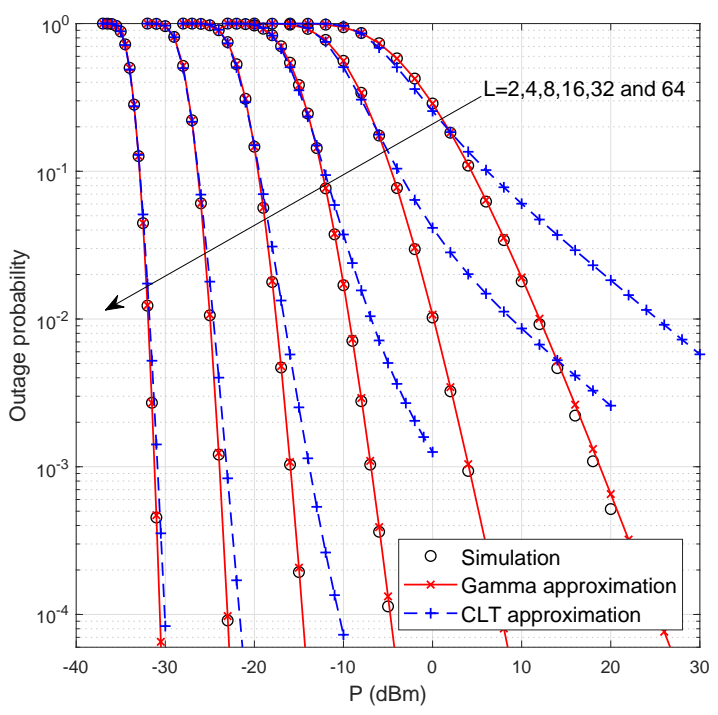

Fig. 5: The outage probability vs $P$ when $\sigma_{i}^{2}=10^{-4}$.

efficiency reduction is around $10.5 \%$ at $P=0 \mathrm{dBm}$ compared Algorithm 1.

Fig. $8 \mathrm{~b}$ plots the spectral efficiency vs transmit power of $U_{1}$ for reciprocal and non-reciprocal channels with different $L$. When $L=1$, both cases show the same spectral efficiency as phase adjustment does not effect the performance. However, when $L \geq 2$, the reciprocal channel case outperforms the nonreciprocal channel case. The reason is that, for each reflective element with reciprocal channels, the effective phase for the SINR is common for both users and the corresponding optimum phase can also maximize the each user SINR. However, for each reflective element with non-reciprocal channels, the effective phases for the SINRs of two users are different and the corresponding optimum phases which maximize the minimum user SINR do not maximize the each user SINR. Therefore, we lose some spectral efficiency compared with reciprocal channel case. As illustrated in Fig. 8b, the spectral efficiency gap between these two cases increases when $L$ increases, e.g., the difference between transmit powers which achieve spectral efficiency 15 [bits/sec/Hz] are $0.3,2.0$ and $6.5 \mathrm{dBm}$ for $L=2,4$ and 16 , respectively.

\section{CONCLUSION}

In this work, RIS assisted systems have been proposed for two-way wireless communications. Two possible transmission schemes are introduced where Scheme 1 and Scheme 2 require one and two resource blocks (time or frequency), respectively. For both reciprocal and non-reciprocal channels, Scheme 1 is the main focus of this work. For the optimal phases of the RIS elements over reciprocal channels, the exact SINR outage probability and average spectral efficiency have been derived for a single-element RIS. Since the exact performance analysis for a multiple-element RIS seems intractable, approximations have been derived for the outage probability and average spectral efficiency. In this respect, a product of two Rayleigh random variables approximated by a gamma random variable. Moreover, asymptotic analysis has been conducted for high SINR $\rho$ regime. Our analysis reveals that the outage probability decreases at the rate of $(\log (\rho) / \rho)^{L}$, whereas spectral efficiency increases at the rate of $\log (\rho)$. Moreover, we observe either an outage or spectral efficiency floor caused by transmit power dependent loop interference. Cross over boundary, where Scheme 1 outperforms Scheme 2 and vice versa, has also been approximately derived based on the asymptotic results. For non-reciprocal channels, an optimization problem is formulated, which optimizes the phases of RIS elements 


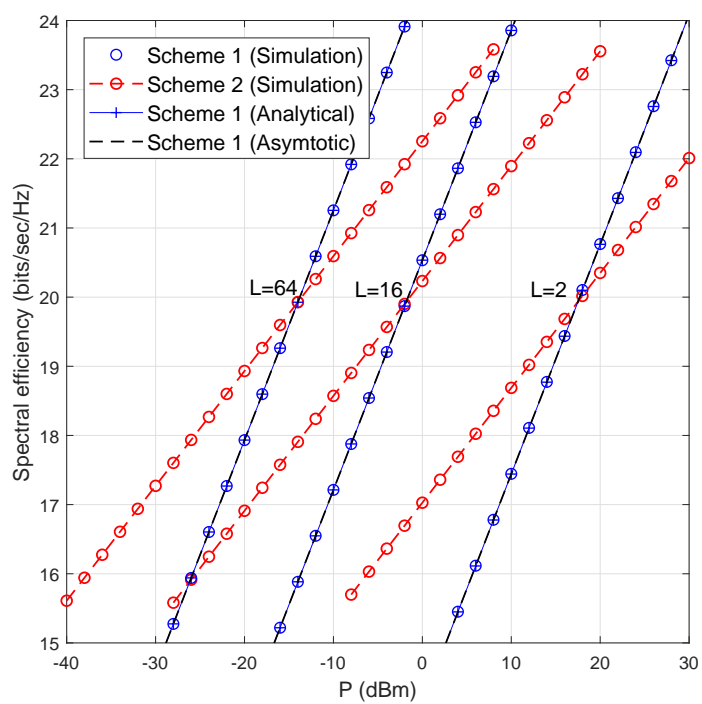

(a) The spectral efficiency vs $P$ when $\sigma_{i}^{2}=\omega=10^{-4}$.

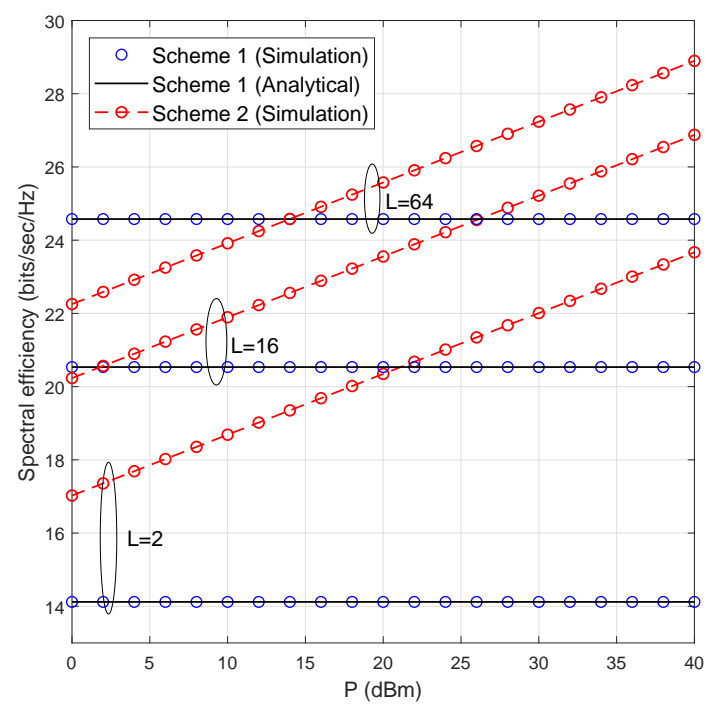

(b) The spectral efficiency vs $P$ when $\sigma_{i}^{2}=\omega P=10^{-4} P$.

Fig. 6: The performance for reciprocal channels with $L \geq 2$.

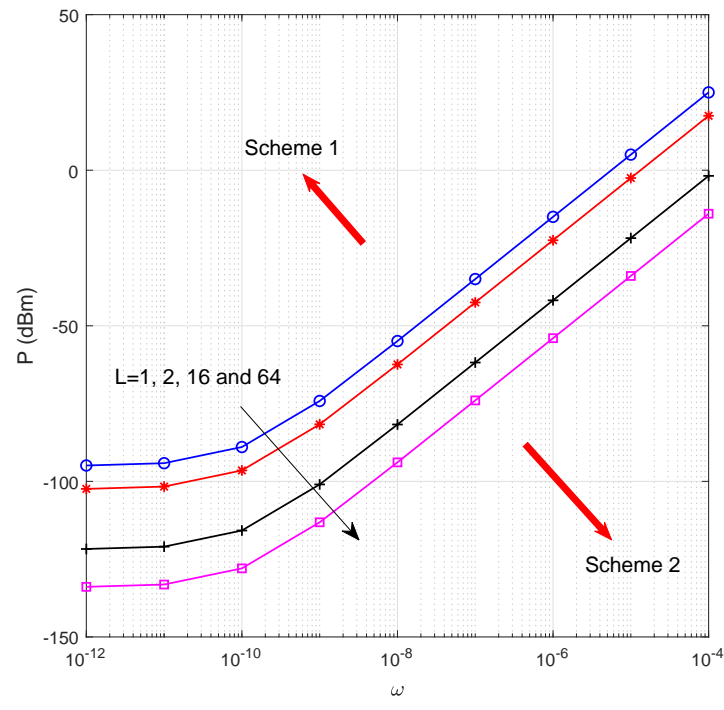

(a) The transmit power vs $n$ of $\sigma_{i}^{2}=\omega=10^{-n}$.

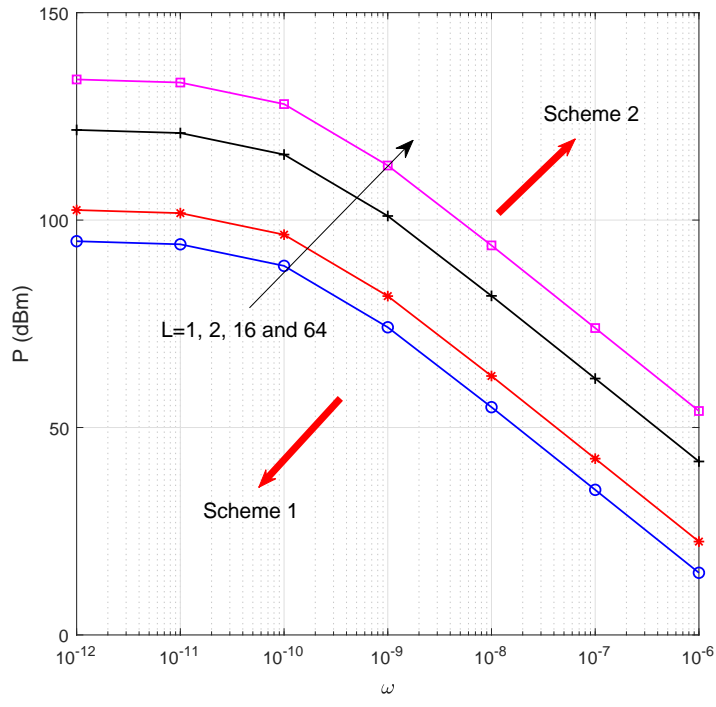

(b) The transmit power vs $n$ of $\sigma_{i}^{2}=\omega P=10^{-n} P$.

Fig. 7: The boundary of transmit power for Scheme 1 and Scheme 2.

so as to maximize the minimum user SINR. Although being non-convex, sub-optimal solution is found by relaxing and then transforming the original optimization problem to be a SDP problem for multiple-element RIS and closed-form solution is found for single-element RIS. Simulation results have illustrated that the rate of spectral efficiency increment or transmit power saving reduces when number of elements increases. A network with reciprocal channels outperforms in terms of outage or spectral efficiency the same with nonreciprocal channels.

\section{APPENDIX A}

PROOF OF THEOREM 1

With the aid of asymptotic expansion of $\mathrm{K}_{1}(x)$ at $x \approx 0$ [28, eq. 8.446], we have, for $a>0$,

$\mathrm{K}_{1}(a \sqrt{x}) \rightarrow \frac{1}{a \sqrt{x}}+\frac{\sqrt{x}}{4}\left(a \log (x)+2 \epsilon a-a+2 a \log \left(\frac{a}{2}\right)\right)$.

For $L=1$, since the outage expression in (16) contains the term $a \sqrt{x} \mathrm{~K}_{1}(a \sqrt{x})$ where $x=1 / \rho$ and $a=\frac{2}{\sigma^{2}} \sqrt{\gamma_{\text {th }}}$, considering the dominant terms, we have a high SINR approximation as $P_{\text {out } \mid \mathrm{L}=1}^{\infty}\left(\gamma_{\text {th }}\right) \rightarrow \frac{\gamma_{\text {th }}}{\sigma^{4}} \frac{\log (\rho)}{\rho}$. 


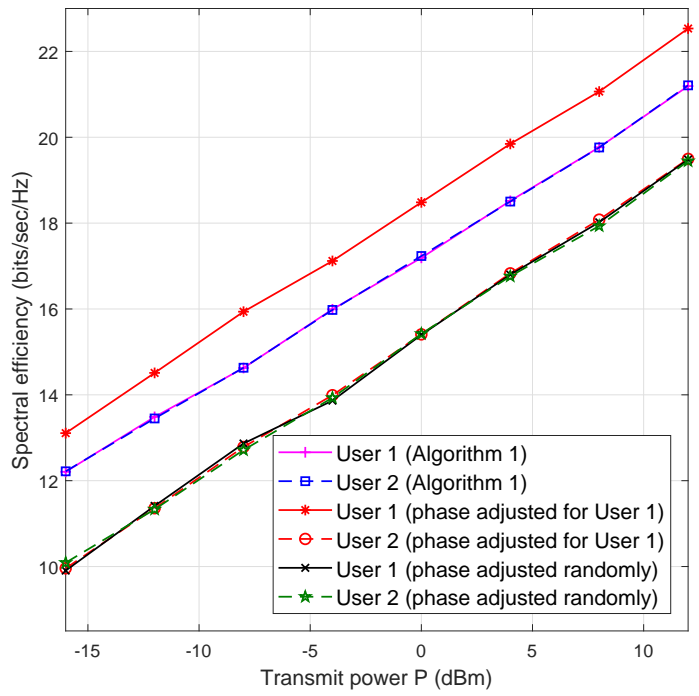

(a) The spectral efficiency vs $P$ for $L=8$.

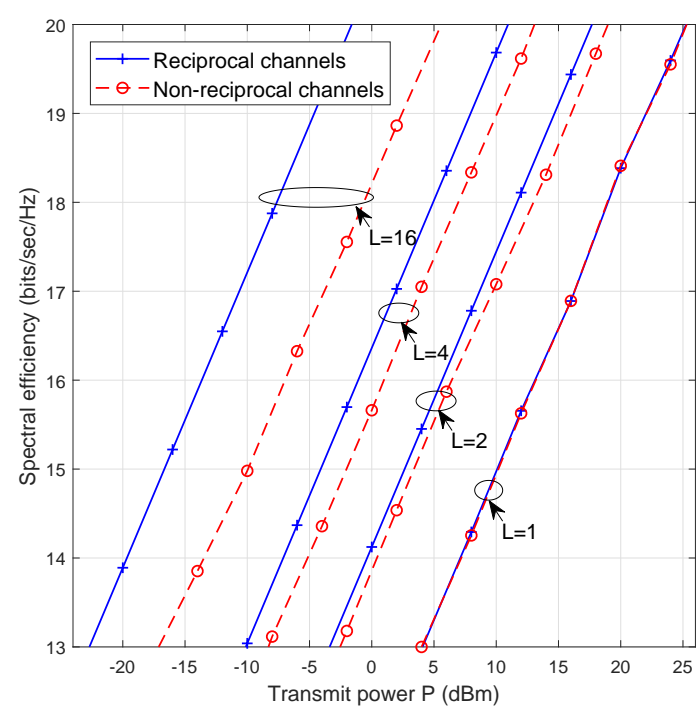

(b) The spectral efficiency vs $P$ for $L=1,2,4,16$.

Fig. 8: The performance of non-reciprocal channels with Scheme 1.

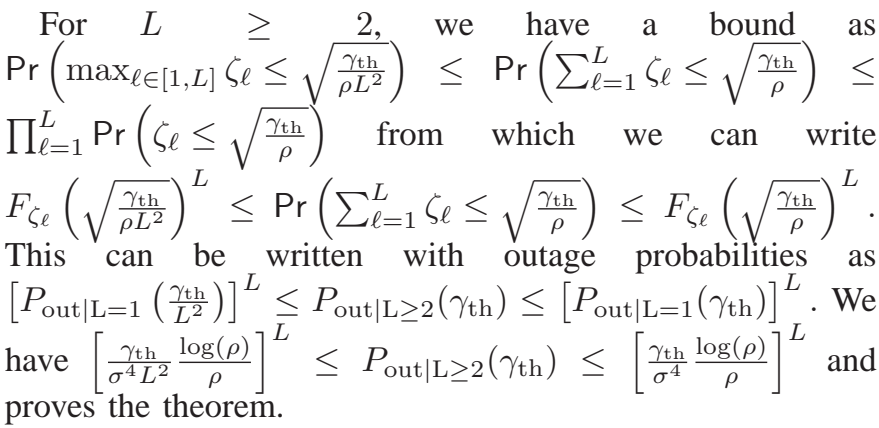

\section{APPENDIX B}

PROOF OF THEOREM 2

We first find the Mellin Transform of $\mathrm{G}_{1,3}^{3,1}(\cdot)$ in (24) by using [35], which gives $-1 / 2 \pi(2 s-1) a^{-s} \sec (\pi s) \Gamma(s-1 / 2)^{2}$ where $a=1 /\left(\sigma^{4} \rho\right)$ and this transform exists within the residue $1 / 2<\operatorname{Re}[s]<3 / 2$. We now sum to the left of the strip starting with $s=1 / 2$ which results

$$
\mathrm{G}_{1,3}^{3,1}\left(\begin{array}{l|c}
a x & -\frac{1}{2} \\
-\frac{1}{2},-\frac{1}{2}, \frac{1}{2}
\end{array}\right) \rightarrow \frac{-\log (a x)-2 \epsilon}{\sqrt{a x}} .
$$

Then, for $L=1$, the average spectral efficiency expression in (24) can be approximated at high SINR, i.e., $\rho \gg$ 1 , as $\mathrm{R}_{\mathrm{L}=1}^{\infty} \rightarrow \frac{\log (\rho)-\log \left(\frac{1}{\sigma^{4}}\right)-2 \epsilon}{\log (2)}$. For $L \geq 2$, with the aid of (25), since the terms associated with hypergeometric functions have negligible effect at $\rho \gg 1$, the average spectral efficiency expression can be approximated as $\mathrm{R}_{\mathrm{L} \geq 2}^{\infty} \rightarrow \frac{\log (\rho)+2 \log (\theta)+2 \psi^{(0)}(L k)}{\log (2)}$. These asymptotic expressions increase at rate $\log (\rho)$ as $\rho$ increases, which proves the theorem.

\section{REFERENCES}

[1] M. D. Renzo et al., "Smart radio environments empowered by AI reconfigurable meta-surfaces: An idea whose time has come," EURASIP J. Wireless Commun. Netw., vol. 2019:129, May 2019.

[2] C. Huang, A. Zappone, G. C. Alexandropoulos, M. Debbah, and C. Yuen, "Reconfigurable intelligent surfaces for energy efficiency in wireless communication," IEEE Trans. Wireless Commun., vol. 18, no. 8, pp. 4157-4170, Aug. 2019.

[3] C. Pan, H. Ren, K. Wang, W. Xu, M. Elkashlan, A. Nallanathan, and L. Hanzo, "Multicell MIMO communications relying on intelligent reflecting surface," Available: https://arxiv.org/abs/1907.10864.

[4] Z. He and X. Yuan, "Cascaded channel estimation for large intelligent metasurface assisted massive mimo," IEEE Wireless Commun. Lett., 2019.

[5] M. Fu, Y. Zhou, and Y. Shi, "Intelligent reflecting surface for downlink non-orthogonal multiple access networks," Available: https://arxiv.org/abs/1906.09434.

[6] C. Pan, H. Ren, K. Wang, M. Elkashlan, A. Nallanathan, J. Wang, and L. Hanzo, "Intelligent reflecting surface aided MIMO broadcasting for simultaneous wireless information and power transfer," Available: https://arxiv.org/abs/1908.04863.

[7] M. Najafi and R. Schober, "Intelligent reflecting surfaces for free space optical communications," Available: https://arxiv.org/abs/1905.01094.

[8] Q. Wu and R. Zhang, "Intelligent reflecting surface enhanced wireless network: Joint active and passive beamforming design," in IEEE Global Telecommn. Conf. (GLOBECOM), Dec. 2018.

[9] X. Yu, D. Xu, and R. Schober, "MISO wireless communication systems via intelligent reflecting surfaces," Available: https://arxiv.org/abs/1904.12199.

[10] Y. Yang, B. Zheng, S. Zhang, and R. Zhang, "MISO wireless communication systems via intelligent reflecting surfaces," Available: https://arxiv.org/abs/1906.09956.

[11] S. Abeywickrama, R. Zhang, and C. Yuen, "Intelligent reflecting surface: Practical phase shift model and beamforming optimization," Available: https://arxiv.org/abs/1907.06002.

[12] H. Guo, Y.-C. Liang, J. Chen, and E. G. Larsson, "Weighted sumrate optimization for intelligent reflecting surface enhanced wireless networks," Available: https://arxiv.org/abs/1905.07920.

[13] Q.-U.-A. Nadeem, A. Kammoun, A. Chaaban, M. Debbah, and M.-S. Alouini, "Asymptotic analysis of large intelligent surface assisted MIMO communication," Available: https://arxiv.org/abs/1903.08127.

[14] M. Cui, G. Zhang, and R. Zhang, "Secure wireless communication via intelligent reflecting surface," IEEE Wireless Commun. Lett., vol. 8, no. 5 , pp. $1410-1414$, Oct. 2019. 
[15] H. Shen, W. Xu, W. Xu, S. Gong, Z. He, and C. Zhao, "Secrecy rate maximization for intelligent reflecting surface assisted multi-antenna communications," IEEE Commun. Lett., vol. 23, no. 9, pp. 1488-1492, Sep. 2019.

[16] Y. Han, W. Tang, S. Jin, C. Wen, and X. Ma, "Large intelligent surfaceassisted wireless communication exploiting statistical CSI," IEEE Trans. Veh. Technol., vol. 68, no. 8, pp. 8238-8242, Aug. 2019.

[17] M. Jung, W. Saad, Y. Jang, G. Kong, and S. Choi, "Performance analysis of large intelligent surfaces (LISs): Asymptotic data rate and channel hardening effects," Available: https://arxiv.org/abs/1810.05667.

[18] E. Basar, M. D. Renzo, J. de Rosny, M. Debbah, M.-S. Alouini, and R. Zhang, "Wireless communications through reconfigurable intelligent surfaces," Available: https://arxiv.org/abs/1906.09490.

[19] M.-A. Badiu and J. P. Coon, "Communication through a large reflecting surface with phase errors," Available: https://arxiv.org/abs/1906.10751.

[20] S. Atapattu, Y. Jing, H. Jiang, and C. Tellambura, "Relay selection schemes and performance analysis approximations for two-way networks," IEEE Trans. Commun., vol. 61, no. 3, pp. 987-998, Mar. 2013.

[21] Z. Zhang, Z. Ma, M. Xiao, G. K. Karagiannidis, Z. Ding, and P. Fan, "Two-timeslot two-way full-duplex relaying for $5 \mathrm{G}$ wireless communication networks," IEEE Trans. Commun., vol. 64, no. 7, pp. 2873-2887, Jul. 2016.

[22] Z. Zhang, Z. Chen, M. Shen, and B. Xia, "Spectral and energy efficiency of multipair two-way full-duplex relay systems with massive MIMO," IEEE J. Select. Areas Commun., vol. 34, no. 4, pp. 848-863, Apr. 2016.

[23] S. Gong, C. Xing, Z. Fei, and S. Ma, "Millimeter-wave secrecy beamforming designs for two-way amplify-and-forward MIMO relaying networks," IEEE Trans. Veh. Technol., vol. 66, no. 3, pp. 2059-2071, Mar. 2017.

[24] P. Ubaidulla and S. Aissa, "Optimal relay selection and power allocation for cognitive two-way relaying networks," IEEE Wireless Commun. Lett., vol. 1, no. 3, pp. 225-228, Jun. 2012.
[25] M. N. Khormuji and M. Skoglund, "On instantaneous relaying," IEEE Trans. Inform. Theory, vol. 56, no. 7, pp. 3378-3394, Jul. 2010.

[26] A. Taha, M. Alrabeiah, and A. Alkhateeb, "Enabling large intelligent surfaces with compressive sensing and deep learning," Available: http://arxiv.org/abs/1904.10136.

[27] L. J. Rodríguez, N. H. Tran, and T. Le-Ngoc, "Performance of fullduplex AF relaying in the presence of residual self-interference," IEEE J. Select. Areas Commun., vol. 32, no. 9, pp. 1752-1764, Sep. 2014.

[28] I. S. Gradshteyn and I. M. Ryzhik, Table of Integrals, Series and Products, 7th ed. Academic Press Inc, 2007.

[29] S. Atapattu, C. Tellambura, and H. Jiang, "A mixture gamma distribution to model the SNR of wireless channels," IEEE Trans. Wireless Commun., vol. 10, no. 12, pp. 4193-4203, Dec. 2011.

[30] A. P. Prudnikov, Y. A. Bryčkov, and O. I. Maričev, Integrals and Series of Special Functions. Moscow, Russia, Russia: Science, 1983.

[31] A. Annamalai, C. Tellambura, and J. Matyjas, "A new twist on the generalized Marcum Q-function $Q_{M}(a, b)$ with fractional-order $M$ and its applications," in IEEE Consumer Commun. Networking Conf. $(C C N C)$, Jan. 2009.

[32] M. Grant and S. Boyd, "CVX: Matlab software for disciplined convex programming, version 2.1," http://cvxr.com/cvx, Dec. 2018

[33] S. Boyd and L. Vandenberghe, Convex Optimization. Cambridge University Press, 2004.

[34] Z. Luo, W. Ma, A. M. So, Y. Ye, and S. Zhang, "Semidefinite relaxation of quadratic optimization problems," IEEE Signal Proc. Mag., vol. 27, no. 3, pp. 20-34, May 2010.

[35] "Mathematica, Version 12.0," http://functions.wolfram.com/ HypergeometricFunctions/MeijerG/06/01/03/01/0003/, accessed: 2019-12-30. 